\title{
ON THE CATEGORY $\mathcal{O}$ FOR RATIONAL CHEREDNIK ALGEBRAS
}

\author{
VICTOR GINZBURG, NICOLAS GUAY, ERIC OPDAM AND RAPHAËL ROUQUIER
}

\begin{abstract}
We study the category $\mathcal{O}$ of representations of the rational Cherednik algebra $A_{W}$ attached to a complex reflection group $W$. We construct an exact functor, called KnizhnikZamolodchikov functor: $\mathcal{O} \rightarrow \mathcal{H}_{W}$-mod, where $\mathcal{H}_{W}$ is the (finite) Iwahori-Hecke algebra associated to $W$. We prove that the Knizhnik-Zamolodchikov functor induces an equivalence between $\mathcal{O} / \mathcal{O}_{\text {tor }}$, the quotient of $\mathcal{O}$ by the subcategory of $A_{W}$-modules supported on the discriminant, and the category of finite-dimensional $\mathcal{H}_{W}$-modules. The standard $\mathrm{A}_{W}$-modules go, under this equivalence, to certain modules arising in Kazhdan-Lusztig theory of "cells", provided $W$ is a Weyl group and the Hecke algebra $\mathcal{H}_{W}$ has equal parameters. We prove that the category $\mathcal{O}$ is equivalent to the module category over a finite dimensional algebra, a generalized " $q$-Schur algebra" associated to $W$.
\end{abstract}

\section{Contents}

1. Introduction

2. Categorv $\mathcal{O}$

2.1. Algebras with triangular decomposition.

2.2. Locallv nilpotent modules

2.3. Standard modules

2.4. Graded modules

2.5. Highest weight theorv

2.6. Properties of categorv $\mathcal{O}$

3. Rational Cherednik algebras

3.1. Basic definitions

3.2. Categorv $\mathcal{O}$ for the rational Cherednik algebra

4. Dualitv. Tiltings. and Projectives

4.1. Ringel dualitv

4.2. Naive dualitv for Cherednik algebras

4.3. Homological properties of Cherednik algebras

5. Hecke algebras via monodromv

5.1. Localisation

5.2. Dunkl operators

5.3. The Knizhnik-Zamolodchikov functor.

5.4. Main results

6. Relation to Kazhdan-Lusztio theorv of cells

6.1. Lusztig's algebra $\mathcal{A}$

6.2. Standard modules for the Hecke algebra via KZ-functor

References

\section{Introduction}

Let $W$ be a complex reflection group acting on a vector space $V$. Let $\mathrm{A}_{W}$ denote the rational Cherednik algebra introduced in [EtGi] as a certain deformation of $\mathcal{D}(V) \rtimes W$, the cross-product 
of $W$ with the algebra of polynomial differential operators on $V$. The algebra $\mathrm{A}_{W}$ can be also realized as an algebra of operators (Dunkl operators) acting on polynomial functions on $V$. When $W$ is a Weyl group, $A_{W}$ is a rational degeneration of the double affine Hecke algebra.

A nice category $\mathcal{O}$ of $\mathrm{A}_{W}$-modules has been discovered in DuOp, cf. also BeEtGi]. It shares many similarities with the Bernstein-Gelfand-Gelfand category $\mathcal{O}$ for a finite-dimensional semisimple Lie algebra.

We develop a general approach to the category $\mathcal{O}$ for a rational Cherednik algebra, similar in spirit to Soergel's analysis, see [So1, of the category $\mathcal{O}$ in the Lie algebra case. Specifically, in addition to the algebra $A_{W}$, we consider an appropriate (finite) Hecke algebra $\mathcal{H}_{W}$, and construct an exact functor $\mathrm{KZ}: \mathcal{O} \rightarrow \mathcal{H}_{W}$-mod, that may be thought of as a Cherednik algebra analogue of the functor $\mathbb{V}$ of [So1]. One of our main results says that the functor KZ is fully faithful on projectives. Thus, the (noncommutative!) Hecke algebra plays, in our case, the role similar to that the coinvariant algebra (= cohomology of the flag manifold) plays in the Lie algebra case. It is also interesting to note that, in both cases, the algebra in question is Frobenius.

To prove our results, in \$2 we develop some basic representation theory over a ground ring (which is not necessarily a field) of a general associative algebra with a triangular decomposition. This generalizes earlier work of the second author $\mathrm{Gu}$ and of the last two authors (unpublished). Such generality will be essential for us in order to use deformation arguments in $\$ 5$. The results of section 2 are applied to Cherednik algebras in $\$ 3.2$.

In 4 , we explain how to generalize some classical constructions for $\mathcal{D}(V)$, the Weyl algebra, (such as characteristic varieties, duality) to the rational Cherednik algebra. We study two kinds of dualities. One of them is related to Fourier transform while the other, much more important one, generalizes the usual (Verdier type) duality on $\mathcal{D}$-modules. This enables us to show that the Ringel dual of category $\mathcal{O}$ is a category $\mathcal{O}$ for the dual reflection group. We also give a formula for the dimension of the characteristic variety involving only the highest weight structure of $\mathcal{O}$.

Our most important results are concentrated in \$5.4. We use the de Rham functor for Knizhnik-Zamolodchikov type $\mathcal{D}$-modules over the complement of the ramification locus in $V$. This way, we relate the category $\mathcal{O}$ with a Hecke algebra. We prove that the category $\mathcal{O}$ can be recovered from its quotient by the subcategory of objects with non-maximal characteristic variety (Theorem [5.3 and Corollary [5.5).

Then, we obtain a "double centralizer" Theorem 5.16, asserting in particular that the category $\mathcal{O}$ is equivalent to the category of modules over the endomorphism ring of some Hecke algebra module. A crucial point is the proof that the de Rham functor sends the $\mathcal{D}$-modules coming from objects of $\mathcal{O}$ to representations of the braid group that factor through the Hecke algebra (Theorem 5.13).

In a different perspective, our results provide a solution to the problem of associating a generalized " $q$-Schur algebra" to an arbitrary finite complex reflection group $W$. This seems to be new even when $W$ is a Weyl group (except for types $\mathbf{A}, \mathbf{B}$ ). For instance, let $W$ be the Weyl group of an irreducible simply-laced root system. Then, the data defining the Cherednik algebra $\mathrm{A}_{W}$ reduces to a single complex parameter $c \in \mathbf{C}$. In this case, $\mathcal{H}_{W}$ is the standard IwahoriHecke algebra of $W$, specialised at the parameter $q=e^{2 \pi i c}$. If $c$ is a rational number, then $q$ is a root of unity, and the corresponding category $\mathcal{H}_{W}$-mod becomes quite complicated. Our results show that the category $\mathcal{O}$ for $A_{W}$ may be viewed as a natural "quasi-hereditary cover" of the 
category $\mathcal{H}_{W}$-mod, which is not itself quasi-hereditary. As a consequence, the decomposition matrices of Hecke algebras (in characteristic 0) are triangular (Corollary 5.19). We remark that, in view of [CPS2, one might have expected on general grounds that the category $\mathcal{H}_{W}$-mod only has a "stratified cover", which is weaker than having a "quasi-hereditary cover".

The reader should be reminded that, in type A, a well-known "quasi-hereditary cover" of $\mathcal{H}_{W}$-mod is provided by the $q$-Schur algebra. We expect that the latter category is equivalent to the category $\mathcal{O}$. Furthermore, for an arbitrary finite Weyl group $W$, we prove in $₫ 6$ that the KZ-functor sends the standard modules in $\mathcal{O}$ to modules over the Hecke algebra (with equal parameters) that can be described via Kazhdan-Lusztig's theory of cells. It follows in particular that, in type $\mathbf{A}$, the standard modules in category $\mathcal{O}$ go to Specht (or 'dual Specht', depending on the sign of parameter ' $c$ ') $\mathcal{H}_{W}$-modules, introduced in [DJ].

Acknowledgments. The second named author gratefully acknowledges the financial support of the Fonds NATEQ. The third named author was partially supported by a Pioner grant of the Netherlands Organization for Scientific Research (NWO).

\section{Category $\mathcal{O}$}

2.1. Algebras with triangular decomposition. In this section, we assume given an associative algebra $A$ with a triangular decomposition. We study a category $\mathcal{O}(A)$ of $A$-modules, similar to the Bernstein-Gelfand-Gelfand category $\mathcal{O}$ for a complex semi-simple Lie algebra. The main result of this section is Theorem 2.19] below, saying that the category $\mathcal{O}(A)$ is a highest weight category (in the sense of [CPS1]).

Throughout this section 2, let $k_{0}$ be an algebraically closed field and $k$ a commutative noetherian $k_{0}$-algebra.

Let $A$ be a graded $k$-algebra with three graded subalgebras $B, \bar{B}$ and $H$ such that

- $A=\bar{B} \otimes H \otimes B$ as $k$-modules

- $B$ and $\bar{B}$ are projective over $k$

- $B \otimes H=H \otimes B$ and $H \otimes \bar{B}=\bar{B} \otimes H$

- $B=\bigoplus_{i \leq 0} B_{i}, \bar{B}=\bigoplus_{i \geq 0} \bar{B}_{i}$, and $B_{0}=\bar{B}_{0}=k$ and $H \subset A_{0}$.

- $H=k \otimes_{k_{0}} H\left(k_{0}\right)$ where $H\left(k_{0}\right)$ is a finite dimensional semi-simple split $k_{0}$-algebra

- the grading on $A$ is inner, i.e., there exists $\partial \in A_{0}$ such that $A_{i}=\{u \in A \mid \partial u-u \partial=i u\}$.

We denote by $B H$ and $\bar{B} H$ the subalgebras $B \otimes H$ and $\bar{B} \otimes H$. We put $B^{i}=B_{-i}$. We denote by $\operatorname{Irr}\left(H\left(k_{0}\right)\right)$ the set of isomorphism classes of finite dimensional simple $H\left(k_{0}\right)$-modules. We put $\partial=\partial^{\prime}-\partial_{0}$ with $\partial^{\prime} \in \bar{B} \otimes H \otimes B^{>0}$ and $\partial_{0} \in Z(H)$. For $E \in \operatorname{Irr}\left(H\left(k_{0}\right)\right)$, we denote by $c_{E}$ the scalar by which $\partial_{0}$ acts on $k \otimes_{k_{0}} E$.

The theory developped here is closely related to the one developped by Soergel [So2, §3-6] in the case where $\mathfrak{g}$ is a graded Lie algebra with $\mathfrak{g}_{0}$ reductive, $A=\mathcal{U}(\mathfrak{g}), B=\mathcal{U}\left(\mathfrak{g}_{>0}\right), \bar{B}=\mathcal{U}\left(\mathfrak{g}_{<0}\right)$ and $H=\mathcal{U}\left(\mathfrak{g}_{0}\right){ }^{1}$

2.2. Locally nilpotent modules. We denote by $\mathcal{O}^{\text {ln }}$ the full subcategory of the category of $A$-modules consisting of those modules that are locally nilpotent for $B$, i.e., an $A$-module $M$ is in $\mathcal{O}^{\text {ln }}$ if for every $m \in M$, there exists $n \gg 0$ such that $B^{>n} \cdot m=0$. This is a Serre subcategory of the category of $A$-modules.

\footnotetext{
${ }^{1}$ In the Lie algebra case, the algebra $H=\mathcal{U}\left(\mathfrak{g}_{0}\right)$ is not finite dimensional. One then has to restrict oneself to the consideration of $H$-semisimple $A$-modules only. The theory developed below easily extends to such a case.
} 
Remark 2.1. The canonical functor $D^{b}\left(\mathcal{O}^{\ln }\right) \rightarrow D^{b}(A)$ is not faithful in general. Nevertheless, for $i=0,1$, and any $M, M^{\prime} \in \mathcal{O}^{\ln }$, one still has $\operatorname{Ext}_{\mathcal{O}^{\ln }}^{i}\left(M, M^{\prime}\right) \stackrel{\sim}{\rightarrow} \operatorname{Ext}_{A}^{i}\left(M, M^{\prime}\right)$.

\subsection{Standard modules.}

2.3.1. Let $h \in H$. We denote by $\phi_{h}: \bar{B} \rightarrow \bar{B} \otimes H \subseteq A$ the map defined by $\phi_{h}(\bar{b})=h \otimes \bar{b}$. Similarly, we denote by $\psi_{h}: B \rightarrow H \otimes B \subseteq A$ the map defined by $\psi_{h}(b)=b \otimes h$.

Let $E$ be an $H$-module. The augmentation $B \rightarrow B / B^{>0}=k$ induces a morphism of algebras $B H \rightarrow H$ and we view $E$ as a $B H$-module by restriction via this morphism. All simple $B H$ modules that are locally nilpotent over $B$ are obtained by this construction, starting with $E$ a simple $H$-module.

We put

$$
\Delta(E)=\operatorname{Ind}_{B H}^{A} E=A \otimes_{B H} E .
$$

The canonical isomorphism $\Delta(E) \stackrel{\sim}{\rightarrow} \bar{B} \otimes E$ is an isomorphism of graded $\bar{B} H$-modules $(E$ is viewed in degree 0 ), where $\bar{B}$ acts by multiplication on $\bar{B}$ and the action of $h \in H$ is given by $\phi_{h} \otimes_{H} 1_{E}: \bar{B} \otimes E \rightarrow \bar{B} \otimes H \otimes_{H} E=\bar{B} \otimes E$.

We now put $\nabla(E)=\operatorname{Homgr}_{\bar{B} H}^{\bullet}(A, E)=\bigoplus_{i} \operatorname{Homgr}_{\bar{B} H}^{i}(A, E)$ (this is also the submodule of elements of $\operatorname{Hom}_{\bar{B} H}(A, E)$ that are locally finite for $\left.B\right)$. Here, $E$ is viewed as a $\bar{B} H$-module via the canonical morphism $\bar{B} H \rightarrow\left(\bar{B} / \bar{B}_{>0}\right) \otimes H=H$.

We have an isomorphism of graded $B H$-modules $\nabla(E) \stackrel{\sim}{\rightarrow} \operatorname{Hom}_{k}(B, k) \otimes E$ where $B$ acts by left multiplication on $\operatorname{Hom}_{k}(B, k)$ and the action of $h \in H$ is given by $f \otimes e \mapsto(b \otimes e \mapsto$ $\left.(1 \otimes f)\left(\psi_{h}(b)\right) e\right)$.

The $A$-module $\Delta(E)$ is a graded module, generated by its degree 0 component. The $A$ module $\nabla(E)$ is also graded. Both $\Delta(E)$ and $\nabla(E)$ are concentrated in non-negative degrees, hence are locally nilpotent for $B$.

\subsubsection{We have}

$$
\operatorname{Ext}_{A}^{i}(\Delta(E), \nabla(F)) \simeq \operatorname{Ext}_{\bar{B} H}^{i}\left(\operatorname{Res}_{\bar{B} H} \Delta(E), F\right) \simeq \operatorname{Ext}_{\bar{B} H}^{i}\left(\operatorname{Ind}_{H}^{\bar{B} H} E, F\right) \simeq \operatorname{Ext}_{H}^{i}(E, F) .
$$

It follows that, when $k$ is a field and $E, F$ are simple, then

$$
\operatorname{Ext}_{A}^{i}(\Delta(E), \nabla(F))=0 \text { if } i \neq 0 \text { or } E \not F \quad \text { and } \quad \operatorname{Hom}_{A}(\Delta(E), \nabla(E)) \simeq k \text {. }
$$

Let $N$ be any $A$-module. We have

$$
\operatorname{Hom}_{A}(\Delta(E), N) \stackrel{\sim}{\rightarrow} \operatorname{Hom}_{B H}\left(E, \operatorname{Res}_{B H} N\right)
$$

2.3.3. A $\Delta$-filtration for a $A$-module $M$ is a filtration $0=M_{0} \subset M_{1} \subset \cdots \subset M_{n}=M$ with $M_{i+1} / M_{i} \simeq \Delta\left(k \otimes_{k_{0}} E_{i}\right)$ for some $E_{i} \in \operatorname{Irr}\left(H\left(k_{0}\right)\right)$. We denote by $\mathcal{O}^{\Delta}$ the full subcategory of $\mathcal{O}^{\text {ln }}$ of objects with a $\Delta$-filtration.

Given an $H$-module $E$ and $n \geq 0$, we also consider more general modules

$$
\Delta_{n}(E)=\operatorname{Ind}_{B H}^{A}\left(\left(B / B^{>n}\right) \otimes_{k} E\right)
$$

The modules $\Delta_{n}\left(k \otimes_{k_{0}} F\right)$ have a $\Delta$-filtration, when $F$ is a finite dimensional $H\left(k_{0}\right)$-module.

For $N$ a $A$-module, we have

$$
\operatorname{Hom}_{A}\left(\Delta_{n}(E), N\right) \stackrel{\sim}{\rightarrow} \operatorname{Hom}_{B H}\left(\left(B / B^{>n}\right) \otimes_{k} E, N\right) .
$$

As a consequence, we have a characterization of $B$-locally nilpotent $A$-modules :

Proposition 2.2. Let $N$ be a A-module. Then, the following are equivalent 
- $N$ is in $\mathcal{O}^{\ln }$

- $N$ is a quotient of a (possibily infinite) sum of $\Delta_{n}(E)$ 's

- $N$ has an ascending filtration whose successive quotients are quotients of $\Delta(E)$ 's.

\subsection{Graded modules.}

2.4.1. Given $\alpha \in k$ and $M$ a $A$-module, define generalized weight spaces in $M$ by

$$
\mathcal{W}_{\alpha}(M)=\left\{m \in M \mid(\partial-\alpha)^{n} m=0 \text { for } n \gg 0\right\} .
$$

Let $\mathcal{O}$ be the full subcategory of $\mathcal{O}^{\text {ln }}$ consisting of those modules $M$ such that $M=\sum_{\alpha \in k} \mathcal{W}_{\alpha}(M)$ where $\mathcal{W}_{\alpha}(M)$ is finitely generated over $k$, for every $\alpha \in k$. This is a Serre subcategory of the category of $A$-modules.

Let $\tilde{\mathcal{O}}$ be the category of graded $A$-modules that are in $\mathcal{O}$. This is a Serre subcategory of the category of graded $A$-modules.

Let $\tilde{\mathcal{O}}^{\alpha}$ be the full subcategory of $\tilde{\mathcal{O}}$ consisting of those objects $M$ such that $M_{i} \subseteq \mathcal{W}_{i-\alpha}(M)$ for all $i$. Note that this amounts to requiring that $\partial^{\prime}-\left(i+c_{F}-\alpha\right)$ acts nilpotently on $\operatorname{Homgr}_{H}^{i}\left(k \otimes_{k_{0}} F, M\right)$ for $F \in \operatorname{Irr}\left(H\left(k_{0}\right)\right)$, since $\partial$ and $\partial_{0}$ commute.

More generally, if $I$ is a subset of $k$, we denote by $\tilde{\mathcal{O}}^{I}$ the full subcategory of $\tilde{\mathcal{O}}$ consisting of those objects $M$ such that $M_{i} \subseteq \sum_{\alpha \in I} \mathcal{W}_{i-\alpha}(M)$.

We denote by $\tilde{\Delta}(E)$ the graded version of $\Delta(E)$ (it is generated in degree 0 and has no terms in negative degrees). Further, write $\langle r\rangle$ for 'grading shift by $r$ ' of a graded vector space.

Lemma 2.3. Let $E \in \operatorname{Irr}\left(H\left(k_{0}\right)\right)$. We have $\tilde{\Delta}\left(k \otimes_{k_{0}} E\right)\langle r\rangle \in \tilde{\mathcal{O}}^{c_{E}-r}$.

Proof. Note that $\partial^{\prime}$ acts as zero on $\tilde{\Delta}\left(k \otimes_{k_{0}} E\right)_{0}$, since $B^{>0}$ acts as zero on it. So, $\partial$ acts as $-c_{E}$ on it. It follows that $\partial$ acts by $i-c_{E}$ on $\bar{B}_{i} \tilde{\Delta}\left(k \otimes_{k_{0}} E\right)_{0}=\tilde{\Delta}\left(k \otimes_{k_{0}} E\right)_{i}$ and we are done.

2.4.2. Let $\mathcal{P}$ be the quotient of $\bigcup_{E \in \operatorname{Irr}\left(H\left(k_{0}\right)\right)}\left(c_{E}+\mathbf{Z}\right)$ by the equivalence relation given as the transitive closure of the relation : $\alpha \sim \beta$ if $\alpha-\beta$ is not invertible.

We make the following assumption until the end of $\$ 2.4$

Hypothesis 1. We assume that $c_{E} \sim c_{E}+n$ for some $n \in \mathbf{Z}$ implies $n=0$ (this holds for example when $k$ is a local ring of characteristic zero).

Proposition 2.4. We have $\tilde{\mathcal{O}}=\bigoplus_{a \in \mathcal{P}} \tilde{\mathcal{O}}^{a}$.

The image by the canonical functor $\tilde{\mathcal{O}} \rightarrow \mathcal{O}$ of $\tilde{\mathcal{O}}^{a+n}$ is a full subcategory $\mathcal{O}^{a+\mathbf{Z}}$ independent of $n \in \mathbf{Z}$.

We have $\mathcal{O}=\bigoplus_{a \in \mathcal{P} / \mathbf{Z}} \mathcal{O}^{a+\mathbf{Z}}$ and the forgetful functor $\tilde{\mathcal{O}}^{a} \stackrel{\sim}{\rightarrow} \mathcal{O}^{a+\mathbf{Z}}$ is an equivalence.

Proof. Let $M$ be an object of $\mathcal{O}$. Let $a \in \mathcal{P}$ and $M^{a}=\sum_{\alpha \in-a+\mathbf{Z}} \mathcal{W}_{\alpha}(M)$. By Lemma 2.3 and Proposition 2.2, we have a decomposition $M=\bigoplus_{a \in \mathcal{P} / \mathbf{Z}} M^{a}$ as $A$-modules.

Similarly, given $\tilde{M} \in \tilde{\mathcal{O}}$, we have $\tilde{M}=\bigoplus_{a \in \mathcal{P}} \tilde{M}^{a}$ where

$$
\tilde{M}^{a}=\bigoplus_{i} \sum_{\alpha \in a}\left(\mathcal{W}_{i-\alpha}(M) \cap M_{i}\right) \in \tilde{\mathcal{O}}^{a} .
$$

Given $M \in \mathcal{O}^{a+\mathbf{Z}}$, we put a grading on $M$ by setting $M_{i}=\sum_{\alpha \in i-a} \mathcal{W}_{\alpha}(M)$ (here we use the assumption on $k$ ). This defines an element of $\tilde{\mathcal{O}}^{a}$ and completes the proof of the proposition.

We denote by $p_{a}: \tilde{\mathcal{O}} \rightarrow \tilde{\mathcal{O}}^{a}$ the projection functor. 
2.4.3. We now give a construction of projective objects (under Hypothesis 1).

Lemma 2.5. Let $a \in \mathcal{P}$ and $d \in \mathbf{Z}$. There is an integer $r$ such that the canonical map

$$
\operatorname{Hom}\left(\tilde{\Delta}_{m}(H)\langle-d\rangle, M\right) \rightarrow M_{d}
$$

is an isomorphism for all $m \geq r$ and $M \in \tilde{\mathcal{O}}^{a}$.

Proof. Replacing $M$ by $M\langle d\rangle$ and $a$ by $a+d$, we can assume that $d=0$.

There is an integer $r$ such that $p_{a}\left(\tilde{\Delta}(H)\left\langle r^{\prime}\right\rangle\right)=0$ for $r^{\prime} \geq r$. The exact sequence

$$
0 \rightarrow \tilde{\Delta}\left(B^{m} \otimes H\right)\langle m\rangle \rightarrow \tilde{\Delta}_{m}(H) \rightarrow \tilde{\Delta}_{m-1}(H) \rightarrow 0
$$

shows that the canonical map

$$
\operatorname{Hom}\left(\tilde{\Delta}_{r}(H), M\right) \stackrel{\sim}{\rightarrow} \operatorname{Hom}\left(\tilde{\Delta}_{m}(H), M\right)
$$

is an isomorphism for any $M \in \tilde{\mathcal{O}}^{a}$ and $m \geq r$. Equivalently, the canonical map

$$
\operatorname{Hom}_{B}\left(B / B^{\geq r}, M\right) \stackrel{\sim}{\rightarrow} \operatorname{Hom}_{B}\left(B / B^{\geq m}, M\right)
$$

is an isomorphism. Since $M$ is locally $B$-nilpotent, this gives an isomorphism

$$
\operatorname{Hom}\left(\tilde{\Delta}_{m}(H), M\right) \stackrel{\sim}{\rightarrow} M_{0} .
$$

Corollary 2.6. Let $E \in \operatorname{Irr}\left(H\left(k_{0}\right)\right)$ and $a \in c_{E}+\mathbf{Z}$. Then, the object $p_{a}\left(\tilde{\Delta}_{r}\left(k \otimes_{k_{0}} E\right)\left\langle a-c_{E}\right\rangle\right)$ of $\tilde{\mathcal{O}}^{a}$ is independent of $r$, for $r \gg 0$. It is projective, has a filtration by modules $\tilde{\Delta}\left(k \otimes_{k_{0}} F\right)\langle r\rangle$ and has a quotient isomorphic to $\tilde{\Delta}\left(k \otimes_{k_{0}} E\right)\left\langle a-c_{E}\right\rangle$.

Corollary 2.7. Let $E \in \operatorname{Irr}\left(H\left(k_{0}\right)\right)$. Then, for $r \gg 0$, the module $\Delta_{r}\left(k \otimes_{k_{0}} E\right)$ has a projective direct summand which is $\Delta$-filtered and has a quotient isomorphic to $\Delta\left(k \otimes_{k_{0}} E\right)$.

Corollary 2.8. There is an integer $r$ such that $\Delta_{r}(H)$ contains a progenerator of $\mathcal{O}$ as a direct summand.

Lemma 2.9. Let $E, F \in \operatorname{Irr}\left(H\left(k_{0}\right)\right)$ such that $\operatorname{Ext}_{\mathcal{O}}^{1}\left(\Delta\left(k \otimes_{k_{0}} E\right), \Delta\left(k \otimes_{k_{0}} F\right)\right) \neq 0$. Then, $c_{F}-c_{E}$ is a positive integer.

Proof. By Lemma 2.3 and Proposition 2.4, we have $\operatorname{Ext}_{\mathcal{O}}^{1}\left(\Delta\left(k \otimes_{k_{0}} E\right), \Delta\left(k \otimes_{k_{0}} F\right)\right)=0$ if $c_{F}-c_{E}$ is not an integer. Assume now $c_{F}-c_{E}$ is an integer. Then

$$
\begin{aligned}
\operatorname{Ext}_{\mathcal{O}}^{1}\left(\Delta\left(k \otimes_{k_{0}} E\right), \Delta\left(k \otimes_{k_{0}} F\right)\right) & \simeq \operatorname{Ext}_{\tilde{\mathcal{O}}}^{1}\left(\tilde{\Delta}\left(k \otimes_{k_{0}} E\right), \tilde{\Delta}\left(k \otimes_{k_{0}} F\right)\left\langle c_{F}-c_{E}\right\rangle\right) \\
& \simeq \operatorname{Ext}_{A}^{1}\left(\tilde{\Delta}\left(k \otimes_{k_{0}} E\right), \tilde{\Delta}\left(k \otimes_{k_{0}} F\right)\left\langle c_{F}-c_{E}\right\rangle\right),
\end{aligned}
$$

by Lemma 2.3 and Proposition 2.4. Now,

$$
\operatorname{Ext}_{A}^{1}\left(\tilde{\Delta}\left(k \otimes_{k_{0}} E\right), \tilde{\Delta}\left(k \otimes_{k_{0}} F\right)\left\langle c_{F}-c_{E}\right\rangle\right) \simeq \operatorname{Ext}_{B H}^{1}\left(k \otimes_{k_{0}} E, \operatorname{Res}_{B H} \tilde{\Delta}\left(k \otimes_{k_{0}} F\right)\left\langle c_{F}-c_{E}\right\rangle\right) .
$$

If the last $\mathrm{Ext}^{1}$ is non zero, then $c_{F}-c_{E}$ is a positive integer.

Corollary 2.10. Assume $k$ is a field. Let $E \in \operatorname{Irr}(H)$. Then, $L(E)$ has a projective cover $P(E)$ with a filtration $Q_{0}=0 \subset Q_{1} \subset \cdots \subset Q_{d}=P(E)$ such that $Q_{i} / Q_{i-1} \simeq \Delta\left(F_{i}\right)$ for some $F_{i} \in \operatorname{Irr}(H), c_{F_{i}}-c_{E}$ is a positive integer for $i \neq d$ and $F_{d}=E$. 
Proof. We know already that there is an indecomposable projective module $P(E)$ as in the statement satisfying all assumptions but the one on $c_{F_{i}}-c_{E}$, by Corollary 2.7.

Take $r \neq d$ maximal such that $Q_{r} / Q_{r-1} \simeq \Delta(F)$ with $c_{F}-c_{E}$ not a positive integer. By Lemma 2.9] the extension of $P(E) / Q_{r-1}$ by $\Delta(F)$ splits. So, we have a surjective morphism $P(E) \rightarrow \Delta(E) \oplus \Delta(F)$. This is impossible since $P(E)$ is indecomposable and projective.

\subsection{Highest weight theory.}

2.5.1. We assume here that $k$ is a field.

For $E$ a simple $H$-module, all proper submodules of $\Delta(E)$ are graded submodules by Proposition 2.4, hence are contained in $\Delta(E)_{>0}$. Consequently, $\Delta(E)$ has a unique maximal proper submodule, hence a unique simple quotient which we denote by $L(E)$.

It follows from (11) that $L(E)$ is the unique simple submodule of $\nabla(E)$ and that $L(E) \nsucceq L(F)$ for $E \not F$.

Proposition 2.11. The simple objects of $\mathcal{O}^{\ln }$ are the $L(E)$ for $E \in \operatorname{Irr}(H)$.

Proof. Let $N \in \mathcal{O}^{\text {ln }}$. Then there is a simple $H$-module $E$ such that $\operatorname{Hom}_{B H}\left(E, \operatorname{Res}_{B H} N\right) \neq 0$. By (2), it follows that every simple object of $\mathcal{O}^{\text {ln }}$ is a quotient of $\Delta(E)$ for some simple $H$-module E.

2.5.2. Let $M$ be a $A$-module. Let $p(M)$ be the set of elements of $M$ annihilated by $B^{>0}$. This is an $H$-submodule of $M$.

Lemma 2.12. Let $M$ be a A-module and $E$ an $H$-module. Then,

- $M$ is a quotient of $\Delta(E)$ if and only if there is a morphism of $H$-modules $\varphi: E \rightarrow p(M)$ such that $M=A \varphi(E)$;

- If $k$ is a field and $E$ is simple, then $M \simeq L(E)$ if and only if $M=A p(M)$ and $p(M) \simeq E$.

In particular, $A p(M)$ is the largest submodule of $M$ that is a quotient of $\Delta(F)$ for some $H$ module $F$.

Proof. The first assertion follows from (2) and the isomorphism

$$
\operatorname{Hom}_{B H}\left(E, \operatorname{Res}_{B H} M\right) \simeq \operatorname{Hom}_{H}(E, p(M)) .
$$

Now, we assume $k$ is a field and $E$ is simple.

Assume $p(M) \simeq E$ and $M=A p(M)$. Then, $M$ is in $\mathcal{O}^{\text {ln }}$. Let $N$ be a non-zero submodule of $M$. We have $0 \neq p(N) \subseteq p(M)$, hence $p(N)=p(M)$ and $N=M$. So, $M$ is simple and isomorphic to $L(E)$ since $M$ is a quotient of $\Delta(E)$.

Assume $M \simeq L(E)$. Since $\operatorname{dim}_{k} \operatorname{Hom}(\Delta(F), L(E))=1$ if $E \simeq F$, and this Hom-space vanishes otherwise, it follows from (2) that $p(M) \simeq E$.

Let $M\{0\}=0$ and define by induction $N\{i\}=M / M\{i\}, L\{i\}=A p(N\{i\})$ and $M\{i+1\}$ as the inverse image of $L\{i\}$ in $M$. We have obtained a sequence of submodules of $M, 0=$ $M\{0\} \subset M\{1\} \subset \cdots \subset M$.

Since $\Delta(E)$ is locally nilpotent for $B$, the following proposition is clear. It describes how the objects of $\mathcal{O}^{\text {ln }}$ are constructed from $\Delta(E)$ 's (cf Proposition 2.2).

Proposition 2.13. A A-module $M$ is locally nilpotent for $B$ if and only if $\bigcup_{i} M\{i\}=M$, i.e., if $M$ has a filtration whose successive quotients are quotients of $\Delta(E)$ 's. 
Lemma 2.14. Assume $k$ is a field. Every A-module quotient $M$ of $\Delta(E)$ has a finite JordanHölder series $0=M^{0} \subset M^{1} \subset \cdots \subset M^{d}=M$ with quotients $M^{i} / M^{i-1} \simeq L\left(F_{i}\right)$ such that $F_{i} \in \operatorname{Irr}(k W), c_{E}-c_{F_{i}}$ is a positive integer for $i \neq d$ and $F_{d}=E$.

Proof. By Proposition 2.4, we can assume $c_{F}-c_{E} \in \mathbf{Z}$. From Proposition 2.4, it follows that $M$ inherits a grading from $\Delta(E)$ (with $M_{0} \neq 0$ and $\left.M_{<0}=0\right)$. Note that since $\partial^{\prime}$ acts as zero on $p(M)$, we have $p(M) \subseteq \bigoplus_{F \in \operatorname{Irr}(H)} M_{c_{E}-c_{F}}$.

We will first show that $M$ has a simple submodule.

Take $i$ maximal such that $p(M)_{i} \neq 0$ and $F$ a simple $H$-submodule of $p(M)_{i}$. Let $L=A F$. Then, $p(L) \subseteq p(M) \cap M_{\geq i}$ and $p(L) \subseteq F \oplus L_{>i}$, hence $p(L)=F$. It follows from Lemma 2.12 that $L \simeq L(F)$ and we are done.

Let $d(M)=\sum_{F \in \operatorname{Irr}(H)} \operatorname{dim} M_{c_{E}-c_{F}}$.

We put $M^{\prime}=M / L$. We have $d\left(M^{\prime}\right)<d(M)$. So, the lemma follows by induction on $d(M)$.

2.6. Properties of category $\mathcal{O}$. We assume here in 2.6 that $k$ is a field. We now derive structural properties of our categories.

2.6.1.

Corollary 2.15. Every object of $\mathcal{O}^{\text {ln }}$ has an ascending filtration whose successive quotients are semi-simple.

Proof. Follows from Lemma 2.14 and Proposition 2.2.

Corollary 2.16. Every object of $\mathcal{O}$ has a finite Jordan-Hölder series.

Proof. The multiplicity of $L(E)$ in a filtration of $M \in \mathcal{O}$ given by Corollary 2.15 is bounded by $\operatorname{dim} \mathcal{W}_{-c_{E}}(M)$, hence the filtration must be finite.

Corollary 2.17. The category $\tilde{\mathcal{O}}^{a}$ is generated by the $L(E)\langle r\rangle$, with $r=c_{E}-a$.

Proof. Follows from Lemma 2.3 and Proposition 2.11.

Corollary 2.18. Given $a \in k$, the full abelian Serre subcategory of the category of A-modules generated by the $L(E)$ with $c_{E} \in a+\mathbf{Z}$ is $\mathcal{O}^{a+\mathbf{Z}}$.

2.6.2.

Theorem 2.19. The category $\mathcal{O}$ is a highest weight category (in the sense of [CPS1] with respect to the relation: $E<F$ if $c_{F}-c_{E}$ is a positive integer.

Proof. Follows from Corollary 2.10] and Lemma 2.14]

The standard and costandard objects are the $\Delta(E)$ and $\nabla(E)$. There are projective modules $P(E)$, injective modules $I(E)$, tilting modules $T(E)$. We have reciprocity formulas, cf. [CPS1, Theorem 3.11]:

$$
[I(E): \nabla(F)]=[\Delta(F): L(E)] \quad \text { and } \quad[P(E): \Delta(F)]=[\nabla(F): L(E)] .
$$

Corollary 2.20. If $c_{E}-c_{F} \notin \mathbf{Z}-\{0\}$ for all $E, F \in \operatorname{Irr}\left(H\left(k_{0}\right)\right)$, then $\mathcal{O}$ is semi-simple.

Proposition 2.21. Let $M \in \mathcal{O}$. The following assertions are equivalent

- $M$ has a $\Delta$-filtration 
- $\operatorname{Ext}_{\mathcal{O}}^{i}(M, \nabla(H))=0$ for $i>0$

- $\operatorname{Ext}_{\mathcal{O}}^{1}(M, \nabla(H))=0$

- the restriction of $M$ to $\bar{B}$ is free.

Proof. The equivalence between the first three assertions is classical. The remaining equivalences follow from the isomorphism $\operatorname{Ext}_{\mathcal{O}}^{1}(M, \nabla(H)) \stackrel{\sim}{\rightarrow} \operatorname{Ext}_{A}^{1}(M, \nabla(H)) \stackrel{\sim}{\rightarrow} \operatorname{Ext}_{\bar{B}}^{1}(M, k)$.

\subsubsection{From Proposition 2.2, we deduce}

Lemma 2.22. Let $M \in \mathcal{O}^{\mathrm{ln}}$. The following conditions are equivalent

- $M \in \mathcal{O}$

- $M$ is finitely generated as a A-module

- $M$ is finitely generated as a $\bar{B}$-module.

Lemma 2.23. There is $r \geq 0$ such that for all $M \in \mathcal{O}, a \in k$ and $m$ in the generalized eigenspace for $\partial^{\prime}$ for the eigenvalue $a$, then $\left(\partial^{\prime}-a\right)^{r} m=0$.

Proof. The action of $\partial^{\prime}$ on $\Delta(H)$ is semi-simple. It follows that, given $r \geq 0, a \in k$ and $m \in \Delta_{r}(H)$ in the generalized eigenspace for $\partial^{\prime}$ for the eigenvalue $a$, then $\left(\partial^{\prime}-a\right)^{r} m=0$.

Now, by Corollary 2.7 there is some integer $r$ such that every object of $\mathcal{O}$ is a quotient of $\Delta_{r}(H)^{l}$ for some $l$.

Proposition 2.24. There is $r \geq 0$ such that every module in $\mathcal{O}^{\ln }$ is generated by the kernel of $B^{\geq r}$. Further, there is an integer $r>0$ such that for $M \in \mathcal{O}^{\ln }$, we have $M\{i\}=M\{r\}$ for $i \geq r$.

Proof. Let $r \geq 0$ such that every projective indecomposable object in $\mathcal{O}$ is a quotient of $\Delta_{r-1}(H)$. This means that every object in $\mathcal{O}$ is generated by the kernel of $B^{\geq r}$. Now, consider $M \in \mathcal{O}^{\text {ln }}$ and $m \in M$. Let $N$ be the $A$-submodule of $M$ generated by $m$. This is in $\mathcal{O}$, hence $m$ is in the submodule of $N$ generated by the kernel of $B^{\geq r}$.

Proposition 2.25. Every object in $\mathcal{O}^{\ln }$ is generated by the 0-generalized eigenspace of $\partial^{\prime}$.

Proof. It is enough to prove the proposition for projective indecomposable objects in $\mathcal{O}$, hence for $\Delta_{r}$ 's, where it is obvious.

2.6.4. Let $Q$ be a progenerator for $\mathcal{O}\left(\operatorname{cf}\right.$ Corollary 2.8) and $\Gamma=\left(\operatorname{End}_{A} Q\right)^{\text {opp }}$. Then, $\Gamma$ is a finitely generated projective $\mathcal{O}$-module. We have mutually inverse standard equivalences

$$
\operatorname{Hom}(Q,-): \mathcal{O} \stackrel{\sim}{\rightarrow} \Gamma \text {-mod, } \quad Q \otimes_{\Gamma}(-): \Gamma-\bmod \stackrel{\sim}{\rightarrow} \mathcal{O} .
$$

Let now $X$ be a (non-necessarily finitely generated) $\Gamma$-module. Then, $Q \otimes_{\Gamma} X$ is a quotient of $Q^{(I)}$ for some set $I$, where $X$ is a quotient of $\Gamma^{(I)}$. Now, $Q^{(I)}$ is in $\mathcal{O}^{\text {ln }}$. So, the functor $Q \otimes_{\Gamma}(-): \Gamma$-Mod $\rightarrow A$-Mod takes values in $\mathcal{O}^{\ln }$ and we have equivalences

$$
\operatorname{Hom}(Q,-): \mathcal{O}^{\ln } \stackrel{\sim}{\rightarrow} \Gamma-\operatorname{Mod}, \quad Q \otimes_{\Gamma}-: \Gamma-\operatorname{Mod} \stackrel{\sim}{\rightarrow} \mathcal{O}^{\ln } .
$$

\section{Rational Cherednik algebras}

3.1. Basic definitions. Let $V$ be a finite dimensional vector space and $W \subset \mathrm{GL}(V)$ a finite complex reflection group. Let $\mathcal{A}$ be the set of reflecting hyperplanes of $W$. Given $H \in \mathcal{A}$, let $W_{H} \subset W$ be the subgroup formed by the elements of $W$ that fix $H$ pointwise. We choose $v_{H} \in V$ such that $\mathbf{C} v_{H}$ is a $W_{H}$-stable complement to $H$. Also, let $\alpha_{H} \in V^{*}$ be a linear form with kernel $H$. 
Let $k$ be a noetherian commutative $\mathbf{C}$-algebra. The group $W$ acts naturally on $\mathcal{A}$ and on the group algebra $k W$, by conjugation. Let $\gamma: \mathcal{A} \rightarrow k W, H \mapsto \gamma_{H}$, be a $W$-equivariant map such that $\gamma_{H}$ is an element of $k W_{H} \subset k W$ with trace zero, for each $H \in \mathcal{A}$.

Given $\gamma$ as above, one introduces an associative $k$-algebra $\mathrm{A}(V, \gamma)$, the rational Cherednik algebra. It is defined as the quotient of $k \otimes_{\mathbf{C}} T\left(V \oplus V^{*}\right) \rtimes W$, the cross-product of $W$ with $k$-tensor algebra, by the relations

$$
\begin{gathered}
{[\xi, \eta]=0 \text { for } \xi, \eta \in V, \quad[x, y]=0 \text { for } x, y \in V^{*}} \\
{[\xi, x]=\langle\xi, x\rangle+\sum_{H \in \mathcal{A}} \frac{\left\langle\xi, \alpha_{H}\right\rangle\left\langle v_{H}, x\right\rangle}{\left\langle v_{H}, \alpha_{H}\right\rangle} \gamma_{H}}
\end{gathered}
$$

Remark 3.1. Let Refl $\subset W$ denote the set of (pseudo)-reflections. Clearly Refl is an $\operatorname{Ad} W$ stable subset. Giving $\gamma$ as above is equivalent to giving a $W$-invariant function $c:$ Refl $\rightarrow$ $k, g \mapsto c_{g}$ such that $\gamma_{H}=\sum_{g \in W_{H} \backslash\{1\}} c_{g} \cdot g$. One may use the function $c$ instead of $\gamma$, and write $v_{g} \in V$, resp. $\alpha_{g} \in V^{*}$, instead of $v_{H}$, resp. $\alpha_{H}$, for any $g \in W_{H} \backslash\{1\}$. Then the last commutation relation in the algebra $\mathrm{A}(V, \gamma)$ reads:

$$
[\xi, x]=\langle\xi, x\rangle+\sum_{g \in \operatorname{Refl}} c_{g} \cdot \frac{\left\langle\xi, \alpha_{g}\right\rangle\left\langle v_{g}, x\right\rangle}{\left\langle v_{g}, \alpha_{g}\right\rangle} \cdot g
$$

which is essentially the commutation relation used in EtGi]. In case of a Weyl group $W$, in [EtGi, BeEtGi, Gu], the coefficients $c_{\alpha}$ ( $\alpha$ a root) were used instead of the $c_{g}$ 's. Then, $\gamma_{H}=-2 c_{\alpha} g$ for $H$ the kernel of $\alpha$ and $g$ the associated reflection.

Remark 3.2. Put $e_{H}=\left|W_{H}\right|$. Denote by $\varepsilon_{H, j}=\frac{1}{e_{H}} \sum_{w \in W_{H}} \operatorname{det}(w)^{j} w$ the idempotent of $\mathbf{C} W_{H}$ associated to the character $\operatorname{det}_{\mid W_{H}}^{-j}$. Given $\gamma$ as above, there is a unique family $\left\{k_{H, i}=\right.$ $\left.k_{H, i}(\gamma)\right\}_{H \in \mathcal{A} / W, 0 \leq i \leq e_{H}}$ of elements of $k$ such that $k_{H, 0}=k_{H, e_{H}}=0$ and

$$
\gamma_{H}=e_{H} \sum_{j=0}^{e_{H}-1}\left(k_{H, j+1}(\gamma)-k_{H, j}(\gamma)\right) \varepsilon_{H, j}
$$

We observe that $\gamma$ can be recovered from the $k_{H, i}(\gamma)$ 's by the formula

$$
\gamma_{H}=\sum_{w \in W_{H}-\{1\}}\left(\sum_{j=0}^{e_{H}-1} \operatorname{det}(w)^{j} \cdot\left(k_{H, j+1}(\gamma)-k_{H, j}(\gamma)\right)\right) w
$$

This way, we get back to the definition of $\mathrm{DuOp}$.

Introduce free commutative positively graded $k$-algebras $\mathrm{P}=k \otimes_{\mathbf{C}} S\left(V^{*}\right)=\bigoplus_{i \geq 0} \mathrm{P}_{i}$ and $\beta=k \otimes_{\mathbf{C}} S(V)=\bigoplus_{i \geq 0} \beta^{i}$. We have a triangular decomposition $\mathrm{A}=\mathrm{P} \otimes_{k} k W \otimes_{k} \beta$ as $k$-modules [EtGi, Theorem 1.3].

For $H \in \mathcal{A}$, we put

$$
a_{H}(\gamma)=\sum_{i=1}^{e_{H}-1} e_{H} \cdot k_{H, i}(\gamma) \cdot \varepsilon_{H, i} \in k\left[W_{H}\right] \quad \text { and } \quad z(\gamma)=\sum_{H \in \mathcal{A}} a_{H}(\gamma) \in Z(k W)
$$

We denote by $\operatorname{Irr}(k W)$ a complete set of representatives of isomorphism classes of simple $k W$-modules. 
For $E \in \operatorname{Irr}(\mathbf{C} W)$, we denote by $c_{E}=c_{E}(\gamma)$ the scalar by which $z(\gamma)$ acts on $k \otimes_{\mathbf{C}} E$. The elements $a_{H}(\gamma), z(\gamma)$, and $c_{E}(\gamma)$, may be thought of as functions of the coefficients $k_{H, i}=$ $k_{H, i}(\gamma)$ (through their dependence on $\gamma$ ). In particular, it was shown in DuOp, Lemma 2.5] that $c_{E}$, expressed as a function of the $k_{H, i}$ 's, is a linear function with non-negative integer coefficients.

Below, we will often use simplified notations and write $\mathrm{A}$ for $\mathrm{A}(\gamma), k_{H, i}$ for $k_{H, i}(\gamma)$, and $z$ for $z(\gamma)$, etc.

We introduce a grading on A by putting $V^{*}$ in degree 1, $V$ in degree -1 and $W$ in degree 0 (thus, the induced grading on the subalgebra $P \subset A$ coincides with the standard one on $P$, while the induced grading on the subalgebra $B \subset \mathrm{A}$ differs by a sign from the standard one on $\beta) .^{2}$

Let $\mathrm{eu}_{k}=\sum_{b \in \mathcal{B}} b^{\vee} b$ be the "deformed Euler vector field", where $\mathcal{B}$ is a basis of $V$ and $\left\{b^{\vee}\right\}_{b \in \mathcal{B}}$ is the dual basis. We also put eu $=\mathrm{eu}_{k}-z$. The elements $\mathrm{eu}_{k}$ and eu commute with $W$. Note that $\sum_{b}\left[b, b^{\vee}\right]=\operatorname{dim} V+\sum_{H} \gamma_{H}$.

We have

$$
[\mathrm{eu}, \xi]=-\xi \text { and }[\mathrm{eu}, x]=x \text { for } \xi \in V \text { and } x \in V^{*} \text {. }
$$

This shows the grading on $\mathrm{A}$ is "inner", i.e., $\mathrm{A}_{i}=\{a \in \mathrm{A} \mid[\mathrm{eu}, a]=i \cdot a\}$.

\subsection{Category $\mathcal{O}$ for the rational Cherednik algebra.}

3.2.1. We apply now the results of $\oint 2$ in the special case: $A=\mathrm{A}=\mathrm{A}(V, \gamma), B=\beta, \bar{B}=\mathrm{P}$, $H=k W, k_{0}=\mathbf{C}, H\left(k_{0}\right)=\mathbf{C} W, \partial=\mathrm{eu}, \partial^{\prime}=\mathrm{eu}_{k}$ and $\partial_{0}=z$. In particular, we have the category $\mathcal{O}(\gamma):=\mathcal{O}(\mathrm{A}(V, \gamma))$, which was first considered, in the setup of Cherednik algebras, in $\mathrm{DuOp}$.

For any (commutative) algebra map $\psi: k \rightarrow k^{\prime}$, there is a base extension functor $\mathcal{O}(\gamma) \rightarrow$ $\mathcal{O}(\psi(\gamma))$ given by $\mathrm{A}(\psi(\gamma)) \otimes_{\mathrm{A}}(-)$.

3.2.2. Assume $k$ is a field. Since $\mathcal{O}$ and $\tilde{\mathcal{O}}$ have finite global dimension (Theorem 2.19), the Grothendieck group of the category of modules coincides with the Grothendieck group $K_{0}$ of projective modules.

We have a morphism of $\mathbf{Z}$-modules $f: K_{0}(\tilde{\mathcal{O}}) \rightarrow \mathbf{Z}[[q]]\left[q^{-1}\right] \otimes K_{0}(\mathbf{C} W)$ given by taking the graded character of the restriction of the module to $W$ :

$$
M \mapsto \sum_{E \in \operatorname{Irr}(k W)} \sum_{i} q^{i} \operatorname{dim} \operatorname{Hom}_{k W}\left(E, M_{i}\right)[E] .
$$

Set $[\mathrm{P}]:=\sum_{E \in \operatorname{Irr}(k W)} \sum_{i} q^{i} \operatorname{dim} \operatorname{Hom}_{k W}\left(E, \mathrm{P}_{i}\right) \cdot[E]$. This is an invertible element of $\mathbf{Z}[[q]]\left[q^{-1}\right] \otimes$ $K_{0}(\mathbf{C} W)$, and for any $F \in \operatorname{Irr}(k W)$, we have $f([\Delta(F)])=[\mathrm{P}] \cdot[F]$. Since the classes of standard modules generate the $K_{0}$-group, we obtain an isomorphism $\frac{1}{[\mathrm{P}]} f: K_{0}(\tilde{\mathcal{O}}) \stackrel{\sim}{\rightarrow} \mathbf{Z}\left[q, q^{-1}\right] \otimes K_{0}(\mathbf{C} W)$.

Let $k\left[\left(\mathbf{k}_{H, i}\right)_{1 \leq i \leq e_{H}-1}\right]$ be the polynomial ring in the indeterminates $\mathbf{k}_{H, i}$ with $\mathbf{k}_{w(H), i}=\mathbf{k}_{H, i}$ for $w \in W$. We have a canonical evaluation morphism $k\left[\left(\mathbf{k}_{H, i}\right)\right] \rightarrow k$ given by the choice of parameters. Let $\mathfrak{m}$ be the kernel of that morphism, $R$ the completion of $k\left[\left(\mathbf{k}_{H, i}\right)\right]$ at $\mathfrak{m}$, and $K$ the field of fractions of $R$.

We have a decomposition map $K_{0}\left(\mathcal{O}_{K}\right) \stackrel{\sim}{\rightarrow} K_{0}(\mathcal{O})$. It sends $\left[\Delta\left(K \otimes_{\mathbf{C}} E\right)\right]$ to $\left[\Delta\left(k \otimes_{\mathbf{C}} E\right)\right]$.

\footnotetext{
${ }^{2}$ We use superscripts to indicate the standard (non-negative) grading on $B$, and subscripts to denote the gradings on $A$ and $P$. Thus, putting formally $\beta_{-i}:=\beta^{i}$ one recovers compatibility: $\beta_{i}=\beta \cap A_{i}$.
} 
Proposition 3.3. Assume $k$ is a field. Then, $[\Delta(E)]=[\nabla(E)]$ and $[P(E)]=[I(E)]$ for any $k W$-module $E$.

Proof. We first consider the equality $[\Delta(E)]=[\nabla(E)]$. The corresponding statement for $K$ is true, since the category is semi-simple in that case. Hence the modules are determined, up to isomorphism, by their socle (resp. by their head).

The statement for $k$ follows by using the decomposition map.

Now, the equality $[P(E)]=[I(E)]$ follows, using the reciprocity formulas $(\$ 2.6 .2)$.

\section{Duality, Tiltings, and Projectives}

4.1. Ringel duality. We keep the setup of $\$ 2.1$ with $k$ being a field. We make the following two additional assumptions

- We have $\bar{B} \otimes H \otimes B=B \otimes H \otimes \bar{B}=A$;

- The subalgebra $B \subset A$ is Gorenstein (with parameter $n$ ), i.e., there exists an integer $n$ such that

$$
\operatorname{Ext}_{B}^{i}(k, B)= \begin{cases}k & \text { if } i=n \\ 0 & \text { if } i \neq n\end{cases}
$$

The Gorenstein condition implies that, for any $E \in \operatorname{Irr}(H)$, viewed as a $B H$-module via the projection $B H \rightarrow H$, we have $\operatorname{Ext}_{B H}^{i}(E, B H)=0$, for all $i \neq n$; moreover, $\operatorname{Ext}_{B H}^{n}(E, B H)=$ $E^{b}$, where $E^{b}$ is a right $B H$-module such that $\operatorname{dim} E^{b}=\operatorname{dim} E$.

Assume further that the algebra $A$ has finite homological dimension. Thus (see $[\mathrm{Bj}]$ ), there is a well-defined duality functor

$$
R \operatorname{Hom}_{A}(-, A): D^{b}(A-\bmod ) \stackrel{\sim}{\rightarrow} D^{b}\left(A^{\text {opp }}-\bmod \right)^{\text {opp }} .
$$

Furthermore, this functor is an equivalence with inverse $R \operatorname{Hom}_{A^{\text {opp }}}(-, A)$.

The triangular decomposition $A=B \otimes H \otimes \bar{B}$ gives a similar decomposition $A^{\text {opp }}=\bar{B}^{\text {opp }} \otimes$ $H^{\text {opp }} \otimes B^{\text {opp }}$, for the opposite algebras. Therefore, we may consider the category $\mathcal{O}\left(A^{\text {opp }}\right)$ and, for any simple right $H$-module $E^{\prime}$, introduce the standard $A^{\text {opp }}$-module

$$
\Delta^{\mathrm{opp}}\left(E^{\prime}\right):=\operatorname{Ind}_{(B H)^{\mathrm{opp}}}^{A^{\mathrm{opp}}} E^{\prime}=E^{\prime} \otimes_{B H} A,
$$

and also the projective object $P^{\text {opp }}\left(E^{\prime}\right) \in \mathcal{O}\left(A^{\text {opp }}\right)$, the tilting object $T^{\text {opp }}\left(E^{\prime}\right) \in \mathcal{O}\left(A^{\text {opp }}\right)$, etc.

Lemma 4.1. The functor $R \operatorname{Hom}_{A}(-, A[n])$ sends $\Delta(E)$ to $\Delta^{\mathrm{opp}}\left(E^{b}\right)$, for $E$ a finite-dimensional $H$-module.

Proof. Using that $A$ is free as a left $B H$-module, we compute

$$
\operatorname{Ext}_{A}^{i}(\Delta(E), A) \stackrel{\sim}{\rightarrow} \operatorname{Ext}_{B H}^{i}(E, A) \stackrel{\sim}{\rightarrow} \operatorname{Ext}_{B H}^{i}(E, B H) \otimes_{B H} A
$$

We see that this space vanishes for $i \neq n$, and for $i=n$ we get $R \operatorname{Hom}_{A}(\Delta(E), A[n]) \simeq$ $E^{b} \otimes_{B H} A=\Delta^{\mathrm{opp}}\left(E^{b}\right)$.

We would like to use the duality functor $R \operatorname{Hom}_{A}(-, A[n])$ to obtain a functor $D^{b}(\mathcal{O}(A)) \rightarrow$ $D^{b}\left(\mathcal{O}\left(A^{\text {opp }}\right)\right)^{\text {opp }}$. To this end, we will exploit a general result below valid for arbitrary highest weight categories (a contravariant version of Ringel duality [Ri, §6]).

Given an additive category $\mathcal{C}$, let $K^{b}(\mathcal{C})$ be the corresponding homotopy category of bounded complexes in $\mathcal{C}$. 
Proposition 4.2. Let $A$ and $A^{\prime}$ be two quasi-hereditary algebras and $\mathcal{C}=A$-mod, $\mathcal{C}^{\prime}=A^{\prime}$-mod the associated highest weight categories. Let $F$ be a contravariant equivalence between the exact categories of $\Delta$-filtered objects $F: \mathcal{C}^{\Delta} \stackrel{\sim}{\rightarrow}\left(\mathcal{C}^{\prime \Delta}\right)^{\text {opp }}$. Then,

- $\quad F$ restricts to equivalences

$$
\mathcal{C} \text {-proj } \stackrel{\sim}{\rightarrow}\left(\mathcal{C}^{\prime} \text {-tilt }\right)^{\text {opp }} \quad \text { and } \quad \mathcal{C} \text {-tilt } \stackrel{\sim}{\rightarrow}\left(\mathcal{C}^{\prime} \text {-proj }\right)^{\text {opp }}
$$

- The canonical equivalences $K^{b}(\mathcal{C}$-proj $) \stackrel{\sim}{\rightarrow} D^{b}(\mathcal{C})$ and $K^{b}\left(\mathcal{C}^{\prime}\right.$-tilt $) \stackrel{\sim}{\rightarrow} D^{b}\left(\mathcal{C}^{\prime}\right)$, yield an equivalence of derived categories

$$
D: D^{b}(\mathcal{C}) \stackrel{\sim}{\rightarrow} D^{b}\left(\mathcal{C}^{\prime}\right)^{\text {opp }} .
$$

- $\quad$ Let $T=F(A)$, an $\left(A \otimes A^{\prime}\right)$-module. Then, we have $D=R \operatorname{Hom}_{A}(-, T)$ and $D^{-1}=$ $R \operatorname{Hom}_{A^{\prime}}(-, T)$. Via duality $(A$-mod $) \stackrel{\sim}{\rightarrow}\left(A^{\text {opp }}\right)$-mod ${ }^{\text {opp }}$, the functor $D$ identifies $\mathcal{C}^{\text {opp }}$ with the Ringel dual of $\mathcal{C}^{\prime}$.

Proof. Let $M \in \mathcal{C}^{\Delta}$. Then, $M$ is projective if and only if $\operatorname{Ext}^{1}(M, \Delta(E))=0$ for every standard object $\Delta(E)$ of $\mathcal{C}$ (indeed, if $0 \rightarrow M^{\prime} \rightarrow P \rightarrow M \rightarrow 0$ is an exact sequence with $P$ projective, then $M^{\prime}$ is $\Delta$-filtered, hence the sequence splits). The module $F(M)$ is tilting if and only if $\operatorname{Ext}^{1}\left(\Delta\left(E^{\prime}\right), F(M)\right)=0$ for every standard object $\Delta\left(E^{\prime}\right)$ of $\mathcal{C}^{\prime}$. We deduce that $M$ is projective if and only if $F(M)$ is tilting.

So, $F$ restricts to equivalences $\mathcal{C}$-proj $\stackrel{\sim}{\rightarrow}\left(\mathcal{C}^{\prime} \text {-tilt }\right)^{\text {opp }}$ and $\mathcal{C}$-tilt $\stackrel{\sim}{\rightarrow}\left(\mathcal{C}^{\prime} \text {-proj }\right)^{\text {opp }}$.

The last assertions of the Proposition are clear.

We can now apply this construction to the category $\mathcal{O}(A)$. Specifically, Lemma $4.1 \mathrm{im}-$ plies that the functor $R \operatorname{Hom}_{A}(-, A[n])$ restricts to an equivalence $\mathcal{O}(A)^{\Delta} \stackrel{\sim}{\rightarrow}\left(\mathcal{O}\left(A^{\text {opp }}\right)^{\Delta}\right)^{\text {opp }}$. Therefore, using Proposition 4.2 we immediately obtain the following

Proposition 4.3. The functor $R \operatorname{Hom}_{A}(-, A[n])^{\text {opp }}$ restricts to equivalences

$$
\mathcal{O}(A) \text {-proj } \stackrel{\sim}{\rightarrow}\left(\mathcal{O}\left(A^{\text {opp }}\right) \text {-tilt }\right)^{\text {opp }} \quad \text { and } \quad \mathcal{O}(A) \text {-tilt } \stackrel{\sim}{\rightarrow}\left(\mathcal{O}\left(A^{\text {opp }}\right) \text {-proj }\right)^{\text {opp }} \text {. }
$$

The canonical equivalences $K^{b}(\mathcal{O}(A)$-proj $) \stackrel{\sim}{\rightarrow} D^{b}(\mathcal{O}(A))$ and $K^{b}\left(\mathcal{O}\left(A^{\text {opp }}\right)\right.$-tilt $) \stackrel{\sim}{\rightarrow} D^{b}\left(\mathcal{O}\left(A^{\text {opp }}\right)\right)$ induce an equivalence $D: D^{b}(\mathcal{O}(A)) \stackrel{\sim}{\rightarrow} D^{b}\left(\mathcal{O}\left(A^{\text {opp }}\right)\right)^{\text {opp }}$, such that $\Delta(E) \mapsto \Delta^{\mathrm{opp}}\left(E^{\mathrm{b}}\right), P(E) \mapsto$ $T^{\mathrm{opp}}\left(E^{b}\right)$, and $T(E) \mapsto P^{\mathrm{opp}}\left(E^{b}\right)$.

Corollary 4.4. The category $\mathcal{O}\left(A^{\text {opp }}\right)^{\text {opp }}$ is the Ringel dual of $\mathcal{O}(A)$.

4.2. Naive duality for Cherednik algebras. Recall the setup of $\$ 3.2$.

Denote by $(-)^{\dagger}: \mathbf{C} W \stackrel{\sim}{\rightarrow} \mathbf{C} W$ the anti-involution given by $w \mapsto w^{\dagger}:=w^{-1}$ for $w \in W$.

In this section, we compare the algebras $\mathrm{A}=\mathrm{A}(V, \gamma)$ and $\mathrm{A}\left(V^{*}, \gamma^{\dagger}\right)$. This will provide us with means to switch between left and right modules, between $\beta$-locally finite and P-locally finite modules.

The anti-involution $(-)^{\dagger}: \mathbf{C} W \stackrel{\sim}{\rightarrow} \mathbf{C} W$ extends to an isomorphism

$$
(-)^{\dagger}: \mathrm{A}(\gamma) \stackrel{\sim}{\rightarrow} \mathrm{A}\left(\gamma^{\dagger}\right)^{\text {opp }} \quad, \quad V \ni \xi \mapsto-\xi, \quad V^{*} \ni x \mapsto x, \quad W \ni w \mapsto w^{-1}
$$

Remark 4.5. If all pseudo-reflections of $W$ have order 2 , then $\gamma^{\dagger}=\gamma$.

Further, we define an isomorphism of $k$-algebras reversing the gradings by

$$
\begin{gathered}
\varphi: \mathrm{A}(V, \gamma) \stackrel{\sim}{\rightarrow} \mathrm{A}\left(V^{*}, \gamma^{\dagger}\right)^{\text {opp }} \\
V \ni \xi \mapsto \xi, \quad V^{*} \ni x \mapsto x, \quad W \ni w \mapsto w^{-1} .
\end{gathered}
$$


Remark 4.6. When $V$ is self-dual, an isomorphism of $\mathbf{C} W$-modules $F: V \stackrel{\sim}{\rightarrow} V^{*}$ extends to an algebra isomorphism (Fourier transform)

$$
\begin{gathered}
F: \mathrm{A}(V, \gamma) \stackrel{\sim}{\rightarrow} \mathrm{A}\left(V^{*}, \gamma\right) \\
V \ni \xi \mapsto F(\xi), \quad V^{*} \ni x \mapsto-F^{-1}(x), \quad W \ni w \mapsto w .
\end{gathered}
$$

The functor $F_{*}$ restricts to an equivalence $\mathcal{O}(V, \gamma) \stackrel{\sim}{\rightarrow} \mathcal{O}\left(V^{*}, \gamma^{\dagger}\right)$.

4.2.1. Given $M \in \mathcal{O}^{\text {ln }}$, denote by $M^{\vee}$ the $k$-submodule of P-locally nilpotent elements of $\operatorname{Hom}_{k}(M, k)$. This is a right $\mathrm{A}$-module. Via $\varphi_{*}$, this becomes a left $\mathrm{A}\left(V^{*}, \gamma^{\dagger}\right)$-module. If $M$ is graded, then $M^{\vee}=\operatorname{Homgr}_{k}^{\bullet}(M, k)$.

Thus we have defined a functor (analogous of the standard duality on the category $\mathcal{O}$ in the Lie algebra case):

$$
(-)^{\vee}: \mathcal{O}^{\ln }(V, \gamma) \rightarrow \mathcal{O}^{\ln }\left(V^{*}, \gamma^{\dagger}\right)^{\text {opp }}
$$

When $k$ is a field, this functor is an equivalence.

Given a $k W$-module $E$, we use the notation $E^{\vee}=\operatorname{Hom}_{k}(E, k)$ for the dual $k W$-module.

Proposition 4.7. We have $\Delta(E)^{\vee} \stackrel{\sim}{\rightarrow} \nabla\left(E^{\vee}\right)$ for any $k W$-module $E$. If $k$ is a field, then

$$
L(E)^{\vee} \stackrel{\sim}{\rightarrow} L\left(E^{\vee}\right), P(E)^{\vee} \stackrel{\sim}{\rightarrow} I\left(E^{\vee}\right), I(E)^{\vee} \stackrel{\sim}{\rightarrow} P\left(E^{\vee}\right), \nabla(E)^{\vee} \stackrel{\sim}{\rightarrow} \Delta\left(E^{\vee}\right), T(E)^{\vee} \stackrel{\sim}{\rightarrow} T\left(E^{\vee}\right) .
$$

Proof. We have

$$
\operatorname{Homgr}_{k}^{\bullet}\left(\mathrm{A} \otimes_{\beta W} E, k\right) \stackrel{\sim}{\rightarrow} \operatorname{Homgr}_{(\beta W)}^{\bullet \text { opp }}\left(\mathrm{A}, \operatorname{Hom}_{k}(E, k)\right)
$$

and the first part of the proposition follows.

The second assertion follows from the characterization of $L(E)$ (resp. $L\left(E^{\vee}\right)$ ) as the unique simple quotient (resp. submodule) of $\Delta(E)$ (resp. $\nabla\left(E^{\vee}\right)$ ). The other assertions are immediate consequences of the homological characterizations of the objects and/or the existence of suitable filtrations.

Note that the functor $(-)^{\vee}$ restricts to a functor $\mathcal{O}(V, \gamma) \rightarrow \mathcal{O}\left(V^{*}, \gamma^{\dagger}\right)^{\text {opp }}$. When $k$ is a field, it is an equivalence. A compatible choice of progenerators for $\mathcal{O}(V, \gamma)$ and $\mathcal{O}\left(V^{*}, \gamma^{\dagger}\right)$ gives then an isomorphism between the algebra $\Gamma(V)$ for $\mathcal{O}(V, \gamma)$ and the oppposite algebra $\Gamma\left(V^{*}\right)^{\text {opp }}$ for $\mathcal{O}\left(V^{*}, \gamma^{\dagger}\right)\left(\operatorname{cf} \oiiint_{2.6 .4}\right)$.

Corollary 4.8. Let $E$ and $F$ be two simple $k W$-modules. Then, the multiplicity of $\Delta(E)$ in a $\Delta$-filtration of $P(F)$, for $\mathcal{O}(V, \gamma)$, is equal to the multiplicity of $L\left(F^{\vee}\right)$ in a composition series of $\Delta\left(E^{\vee}\right)$, for $\mathcal{O}\left(V^{*}, \gamma^{\dagger}\right)$.

Proof. By $₫ 2.19$, the multiplicity of $\nabla\left(E^{\vee}\right)$ in a $\nabla$-filtration of $I\left(F^{\vee}\right)$ is equal to the multiplicity of $L\left(F^{\vee}\right)$ in a composition series of $\Delta\left(E^{\vee}\right)$.

The functor $(-)^{\vee}$ sends $P(F)$ to $I\left(F^{\vee}\right)$ and $\Delta(E)$ to $\nabla\left(E^{\vee}\right)$ (Proposition 4.7]) and the result follows.

Remark 4.9. When $k$ is a field and $W$ is real, we obtain, via Fourier transform, a duality on $\mathcal{O}^{\text {ln }}$ and on $\mathcal{O}$. Since all complex representations of $W$ are self-dual, we have then $\Delta(E)^{\vee} \stackrel{\sim}{\rightarrow} \nabla(E)$.

\subsection{Homological properties of Cherednik algebras.}


4.3.1. The rational Cherednik algebra is a deformation of the cross-product of $W$ with the Weyl algebra of polynomial differential operators on $V$. In particular, there is a standard increasing filtration on A with $W$ placed in degree 0 and $V \oplus V^{*}$ in degree 1 . The associated graded ring, grA, is isomorphic to $S\left(V \oplus V^{*}\right) \rtimes W$ [EtGi, §1]. It follows (see [Bj], [Bo, $\left.\S \mathrm{V} .2 .2\right]$ ), that $\mathrm{A}$ is left and right noetherian, provided $k$ is noetherian. Since $V^{*} \oplus V$ is a smooth variety of dimension $2 \operatorname{dim} V$, the algebra A has homological dimension at most $2 \operatorname{dim} V$. Furthermore, the usual results and concepts on $\mathcal{D}$-modules (characteristic variety, duality) also make sense for $A$, even though the algebra grA is not commutative.

4.3.2. We assume $k$ is a field, and put $n=\operatorname{dim} V$. The algebras $\beta$ and $\mathrm{P}$ are clearly Gorenstein with parameter $n$. Moreover, we have $\operatorname{Ext}_{\beta}^{n}(k, \beta) \simeq \Lambda^{n} V^{*}$. Hence, $E^{b}=\Lambda^{n} V^{*} \otimes \operatorname{Hom}_{k}(E, k)=$ $\Lambda^{n} V^{*} \otimes E^{\vee}$, for any finite dimensional $W$-module $E$.

It will be useful to compose the functor $R \operatorname{Hom}_{\mathrm{A}(\gamma)}(-, \mathrm{A}(\gamma))$ with the anti-involution $(-)^{\dagger}$, see (5), to get the following composite equivalence

$$
R \operatorname{Hom}_{\mathrm{A}(\gamma)}(-, \mathrm{A}(\gamma))^{\dagger}: D^{b}(\mathrm{~A}(\gamma) \text {-mod }) \stackrel{\sim}{\rightarrow} D^{b}\left(\mathrm{~A}(\gamma)^{\text {opp }} \text {-mod }\right)^{\text {opp }} \stackrel{\sim}{\rightarrow} D^{b}\left(\mathrm{~A}\left(\gamma^{\dagger}\right) \text {-mod }\right)^{\text {opp }} .
$$

From Proposition 4.2 we immediately obtain the following

Proposition 4.10. The functor $R \operatorname{Hom}_{\mathrm{A}}(-, \mathrm{A}[n])^{\dagger}$ gives rise to an equivalence

$$
D: D^{b}(\mathcal{O}(\gamma)) \stackrel{\sim}{\rightarrow} D^{b}\left(\mathcal{O}\left(\gamma^{\dagger}\right)\right)^{\text {opp }} .
$$

We further introduce an equivalence

$$
(-)^{\vee} \circ D: D^{b}(\mathcal{O}(V, \gamma)) \stackrel{\sim}{\rightarrow} D^{b}\left(\mathcal{O}\left(V^{*}, \gamma\right)\right)
$$

such that

$$
\begin{aligned}
\Delta(E) & \mapsto \nabla\left(\Lambda^{n} V \otimes_{\mathbf{C}} E\right) \\
T(E) & \mapsto I\left(\Lambda^{n} V \otimes_{\mathbf{C}} E\right) \\
P(E) & \mapsto T\left(\Lambda^{n} V \otimes_{\mathbf{C}} E\right)
\end{aligned}
$$

In particular, we obtain (cf. Corollary 4.4)

Corollary 4.11. The category $\mathcal{O}\left(V^{*}, \gamma\right)$ is the Ringel dual of $\mathcal{O}(V, \gamma)$.

Remark 4.12. Note that if $W$ is real, then $\mathcal{O}$ is its own Ringel dual.

4.3.3. Semiregular bimodule. Write $\mathrm{P}^{\circledast}=\oplus_{i} \operatorname{Hom}\left(\mathrm{P}_{i}, k\right)$ for the graded dual of $\mathrm{P}$, and form the vector space $R:=\mathbf{P}^{\circledast} \otimes_{k} \beta W$. Let us fix an isomorphism of $\mathbf{C}$-vector spaces $\Lambda^{n} V \stackrel{\sim}{\rightarrow} \mathbf{C}$. We have the following canonical isomorphisms:

$$
\operatorname{Homgr}_{\beta W}^{\bullet}\left(\mathrm{A}, \Lambda^{n} V \otimes_{\mathbf{C}} \beta W\right) \stackrel{\sim}{\rightarrow} \operatorname{Homgr}_{k}^{\bullet}(\mathrm{P}, \beta W) \stackrel{\sim}{\leftarrow} \mathrm{P}^{\circledast} \otimes_{k} \beta W \stackrel{\sim}{\rightarrow} \mathrm{P}^{\circledast} \otimes_{\mathrm{P}} \mathrm{A} .
$$

The first two isomorphisms define a left $\mathrm{A}$-module structure on $R$, and the last one defines a right $\mathrm{A}$-module structure on $R$. It is possible to check by explicit calculations that the left and right $\mathrm{A}$-module structures commute, so that $R$ becomes an A-bimodule. It is a Cherednik algebra analogue of the semiregular bimodule, considered in [A], So2] in the Lie algebra case.

From the isomorphisms of A-modules

$$
R \otimes_{\mathrm{A}} \Delta(E)=R \otimes_{\beta W} E \stackrel{\sim}{\rightarrow} \operatorname{Homgr}_{\beta W}^{\bullet}\left(\mathrm{A}, \Lambda^{n} V \otimes E\right)
$$

we deduce

Proposition 4.13. The functor $M \mapsto \operatorname{Homgr}_{k}^{\bullet}\left(R \otimes_{\mathrm{A}} M, k\right)^{\dagger}$ (= left $\mathrm{A}\left(V, \gamma^{\dagger}\right)$-module) sends $\Delta(E)$ to $\Delta\left(\Lambda^{n} V^{*} \otimes_{\mathbf{C}} E^{\vee}\right)$. 
4.3.4. Given $M$ a finitely generated A-module, a good filtration of $M$ is a structure of filtered A-module on $M$ such that $\operatorname{gr} M$ is a finitely generated grA-module. The characteristic variety $\mathrm{Ch}(M)$ is the support of $\operatorname{gr} M$, viewed as a $W$-equivariant sheaf on $V^{*} \oplus V$ (a closed subvariety). It is well defined, i.e., is independent of the choice of the good filtration (every finitely generated A-module admits a good filtration). Note that Bernstein's inequality: $\operatorname{dim} \operatorname{Ch}(M) \geq \operatorname{dim} V \operatorname{does}$ not hold in general. Further, for $M$ in $\mathcal{O}$, the complex $D(M)$ has zero homology outside the degrees $0, \ldots, n$.

Let $T=\bigoplus_{E} T(E)$ where $E$ runs over the simple $k W$-modules.

Corollary 4.14. Let $M \in \mathcal{O}$. Then, $\operatorname{dim} \operatorname{Ch}(M)=\operatorname{dim} V-\min \left\{i \mid \operatorname{Ext}_{\mathcal{O}}^{i}(T, M) \neq 0\right\}$.

Proof. Let $R=\operatorname{End}(T)^{\text {opp }}$. The functor $R \operatorname{Hom}_{\mathcal{O}}(T,-): D^{b}(\mathcal{O}) \stackrel{\sim}{\rightarrow} D^{b}(R$-mod) is an equivalence. Composing with the inverse of $(-)^{\vee} \circ D$ we obtain an equivalence $D^{b}\left(\mathcal{O}\left(V^{*}, \gamma\right)\right) \stackrel{\sim}{\rightarrow}$ $D^{b}(R$-mod $)$ that restricts to an equivalence $\mathcal{O}\left(V^{*}, \gamma\right) \stackrel{\sim}{\rightarrow} R$-mod. We see that $\min \{i \mid$ $\left.\operatorname{Ext}_{\mathcal{O}}^{i}(T, M) \neq 0\right\}=\min \left\{i \mid H^{i}(D M) \neq 0\right\}$, where the RHS is equal to $\min \left\{i \mid \operatorname{Ext}_{\mathrm{A}}^{i}(M, \mathrm{~A}) \neq\right.$ $0\}-\operatorname{dim} V$ by the definition of $D$. The result now follows from the well-known formula, see e.g Bj: $\operatorname{dim} \operatorname{Ch}(M)=2 \operatorname{dim} V-\min \left\{i \mid \operatorname{Ext}_{\mathrm{A}}^{i}(M, \mathrm{~A}) \neq 0\right\}$.

\section{HeCke Algebras via monodromy}

\subsection{Localisation.}

5.1.1. Let $V_{\text {reg }}=V-\bigcup_{H \in \mathcal{A}} H$ and $\mathrm{P}_{\text {reg }}=k\left[V_{\text {reg }}\right]=\mathrm{P}\left[\left(\alpha_{H}^{-1}\right)\right]_{H \in \mathcal{A}}$. The algebra structure on $\mathrm{A}$ extends to an algebra structure on $\mathrm{A}_{\text {reg }}=\mathrm{P}_{\text {reg }} \otimes_{k} \beta \otimes_{k} k W$.

We denote by

$$
M \mapsto M_{\text {reg }}=\mathrm{A}_{\text {reg }} \otimes_{\mathrm{A}} M: \mathrm{A}-\mathrm{Mod} \rightarrow \mathrm{A}_{\text {reg- }}-\mathrm{Mod}
$$

the localisation functor. Note that $\operatorname{Res}_{\mathrm{P} \text { reg }} M_{\mathrm{reg}}=\mathrm{P}_{\text {reg }} \otimes_{\mathrm{P}} M$. Note also that every element of $M_{\text {reg }}$ can be written as $\alpha^{r} \otimes m$ for some $r \leq 0, m \in M$, where $\alpha=\prod_{H \in \mathcal{A}} \alpha_{H}$. This makes the localisation functor have specially good properties.

The restriction functor $\mathrm{A}_{\text {reg }} \mathrm{Mod} \rightarrow \mathrm{A}-\mathrm{Mod}$ is a right adjoint to the localisation functor. It is fully faithful. The adjunction morphism coincides with the natural localisation morphism $M \rightarrow M_{\text {reg }}$ of A-modules. Its kernel is $M_{\text {tor }}$, the submodule of $M$ of elements whose support is contained in $V-V_{\text {reg. }}$. Denote by (A-Mod) tor the full subcategory of A-Mod of objects $M$ such that $M_{\text {reg }}=0$. The following is clear.

Lemma 5.1. The localisation functor induces an equivalence

$$
\text { A-Mod } /(\text { A-Mod })_{\text {tor }} \stackrel{\sim}{\rightarrow} \mathrm{A}_{\text {reg }}-\operatorname{Mod} \text {. }
$$

The category $\mathcal{O}$ is a Serre subcategory of A-Mod. Let $\mathcal{O}_{\text {tor }}=\mathcal{O} \cap(A-M o d)_{\text {tor }}$. Then, the canonical functor $\mathcal{O} / \mathcal{O}_{\text {tor }} \rightarrow$ A-Mod $/(\mathrm{A}-\mathrm{Mod})_{\text {tor }}$ is fully faithful. Consequently, the canonical functor

$$
\mathcal{O} / \mathcal{O}_{\text {tor }} \rightarrow \mathrm{A}_{\text {reg }}-\mathrm{Mod}
$$

is fully faithful, with image a full abelian subcategory closed under taking subobjects and quotients (but in general not closed under extensions). 
5.1.2. When $k$ is a field, we have a commutative diagram

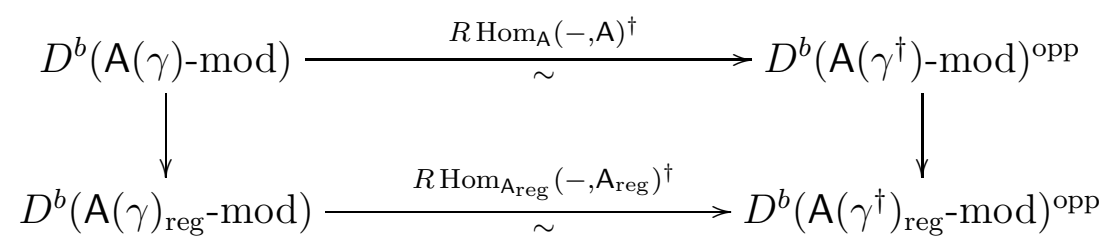

where the vertical arrows are given by localisation.

5.1.3.

Lemma 5.2. Assume $k$ is a field. Then, $(-)^{\vee}$ restricts to an equivalence $\mathcal{O}_{\text {tor }}(V, \gamma) \stackrel{\sim}{\rightarrow}$ $\mathcal{O}_{\text {tor }}\left(V^{*}, \gamma^{\dagger}\right)^{\text {opp }}$.

Proof. Let $M \in \mathcal{O}$. We put a grading on $M$ (Proposition 2.4). Since $M$ is a finitely generated graded P-module (Lemma 2.22), the dimension of $\mathrm{Ch}(M)$, the characteristic variety of $M$, can be obtained from the growth of the function $i \mapsto \operatorname{dim} M_{i}$. In particular, $M \in \mathcal{O}_{\text {tor }}$ if and only if $\lim _{i \rightarrow \infty}\left(i^{1-\operatorname{dim} V} \cdot \operatorname{dim} M_{i}\right)=0$. Such a property is preserved by $(-)^{\vee}$.

Denote by $\mathbb{V}: \mathcal{O} \rightarrow \overline{\mathcal{O}}=\mathcal{O} / \mathcal{O}_{\text {tor }}, M \mapsto \bar{M}$, the quotient functor (the notation $\mathbb{V}$ has been used by Soergel [So1] for an analogous functor in the Lie algebra setup). The functor $\mathbb{V}$ admits, by the standard 'abstract nonsense', both a left adjoint and right adjoint functors ${ }^{\top} \mathbb{V}, \mathbb{V}^{\top}: \overline{\mathcal{O}} \rightarrow \mathcal{O}$.

Theorem 5.3. Assume $k$ is a field, and $Q$ is a projective in $\mathcal{O}$. Then, the canonical adjunction morphism $a: Q \rightarrow \mathbb{V}^{\top}(\bar{Q})$ is an isomorphism. In particular, for any object $M$ in $\mathcal{O}$, the following canonical morphism is an isomorphism

$$
\mathbb{V}_{*}: \operatorname{Hom}_{\mathcal{O}}(M, Q) \stackrel{\sim}{\rightarrow} \operatorname{Hom}_{\overline{\mathcal{O}}}(\bar{M}, \bar{Q}) .
$$

Proof. By $\$ 5.1 .1$, for any two objects $M, Q$, of $\mathcal{O}$, we have a canonical isomorphism

$$
\operatorname{Hom}_{\overline{\mathcal{O}}}(\bar{M}, \bar{Q}) \stackrel{\sim}{\rightarrow} \operatorname{Hom}_{\mathrm{A}_{\mathrm{reg}}}\left(M_{\mathrm{reg}}, Q_{\mathrm{reg}}\right) .
$$

Assume $Q$ has a $\Delta$-filtration. Then it is free over $\mathrm{P}$ and thus has no non-zero submodule lying in $\mathcal{O}_{\text {tor }}$, hence $\mathbb{V}_{*}$ is injective.

Assume furthermore that $M$ has a $\nabla$-filtration. Then $M^{\vee}$ has a $\Delta$-filtration (Proposition 4.7), hence has no non-zero submodule lying in $\mathcal{O}_{\text {tor }}$. Since $(-)^{\vee}$ restricts to an equivalence $\mathcal{O}_{\text {tor }}(V, \gamma) \stackrel{\sim}{\rightarrow} \mathcal{O}_{\text {tor }}\left(V^{*}, \gamma^{\dagger}\right)^{\text {opp }}$ (Lemma 5.2), it follows that $M$ has no non-zero quotient lying in $\mathcal{O}_{\text {tor }}$. This shows that $\mathbb{V}_{*}$ in (8) is an isomorphism.

From now on, we assume that $Q$ is projective. It follows that $Q^{\prime}=D(Q)$ is tilting (Proposition 4.10), hence $\nabla$-filtered.

Now let $M$ be a $\Delta$-filtered object. Then, $M^{\prime}=D(M)$ is $\Delta$-filtered. We apply the result on $\mathbb{V}_{*}$, that we have already proved, to $\mathcal{O}\left(\gamma^{\dagger}\right)$. This yields, by duality (cf \$5.1.2), that (8) is an isomorphism, for any $\Delta$-filtered object $M$.

Since any projective is $\Delta$-filtered, for any two projective objects $P, Q$ in $\mathcal{O}$, we have established the isomorphisms

$$
\operatorname{Hom}_{\mathcal{O}}(P, Q) \stackrel{\stackrel{\mathbb{V}_{*}}{\rightarrow}}{\rightarrow} \operatorname{Hom}_{\overline{\mathcal{O}}}(\bar{P}, \bar{Q}) \stackrel{\text { adjunction }}{=} \operatorname{Hom}_{\mathcal{O}}\left(P, \mathbb{V}^{\top}(\bar{Q})\right)
$$


The above isomorphisms imply, in particular, that, for any indecomposable projective $P$, we have $\operatorname{dim} \operatorname{Hom}_{\mathcal{O}}(P, Q)=\operatorname{dim}_{\operatorname{Hom}_{\mathcal{O}}}\left(P, \mathbb{V}^{\top}(\bar{Q})\right)$. It follows readily that the objects $Q$ and $\mathbb{V}^{\top}(\bar{Q})$ have the same composition factors with the same multiplicities.

We can finally prove that the canonical adjunction map $a: Q \rightarrow \mathbb{V}^{\top}(\bar{Q})$ is an isomorphism. By the previous paragraph, it suffices to show that $a$ is injective. To this end, put $K:=\operatorname{ker}(a)$, and assume $K \neq 0$. Let $L(E)$ be a simple submodule in $K$, and $P(E) \rightarrow L(E)$, its projective cover. By construction, the composite map $g: P(E) \rightarrow L(E) \hookrightarrow K \hookrightarrow Q$ is nonzero. This map $g \in \operatorname{Hom}_{\mathcal{O}}(P(E), Q)$ goes, under the isomorphism between the left-hand and right-hand sides of (9)), to the map $a \circ g: P(E) \rightarrow K \hookrightarrow Q \stackrel{a}{\rightarrow} \mathbb{V}^{\top}(\bar{Q})$. But the latter map is the zero map since $K=\operatorname{ker}(a)$, which contradicts the fact that (9) is an isomorphism. Thus, $\operatorname{ker}(a)=0$, and the Theorem is proved.

Remark 5.4. In general, the assumption that $Q$ is projective cannot be replaced by the weaker assumption that it is $\Delta$-filtered (already for $W=\mathbf{Z} / 2 \mathbf{Z}$ ). Nevertheless, see Proposition 5.9.

Corollary 5.5. Let $X$ be a progenerator of $\mathcal{O}$ and $\mathcal{E}:=\left(\operatorname{End}_{\overline{\mathcal{O}}} \bar{X}\right)^{\mathrm{opp}}$. Then there is an equivalence $\mathcal{O} \stackrel{\sim}{\rightarrow}(\mathcal{E} \text {-mod })^{\mathrm{opp}}$.

Proof. The preceding theorem implies that $\left(\operatorname{End}_{\mathcal{O}} X\right)^{\text {opp }} \stackrel{\sim}{\rightarrow} \mathcal{E}$ since projective modules are $\Delta$-filtered. Hence we can use category equivalences (3).

\subsection{Dunkl operators.}

5.2.1. One has an $A$-action on the vector space $P$, hence an $B$-action, arising via the identification $\mathrm{P}=\Delta(k)$. One finds, in particular, that the action of $\xi \in V$ on $\mathrm{P}$ is given by the Dunkl operator

$$
T_{\xi}=\partial_{\xi}+\sum_{H \in \mathcal{A}} \frac{\left\langle\xi, \alpha_{H}\right\rangle}{\alpha_{H}} \cdot a_{H} \in \mathcal{D}\left(V_{\text {reg }}\right) \rtimes W,
$$

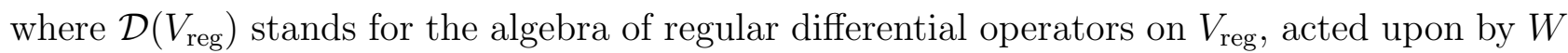
in a natural way, and $a_{H} \in k W$ is viewed as an element of $\mathcal{D}\left(V_{\text {reg }}\right) \rtimes W$. It follows that $T_{\xi}(\mathrm{P}) \subset \mathrm{P}$ (as part of $\mathrm{A}$-action on $\mathrm{P}=\Delta(k)$ ); furthermore, this A-action on $\mathrm{P}$ is known (Cherednik, EtGi, Proposition 4.5]) to be faithful:

Theorem 5.6. The A-representation $\Delta(k)$ is faithful. Thus, the natural action of $\mathrm{P} W$ on $\mathrm{P}$ extends to an injective algebra morphism $i: \mathrm{A} \hookrightarrow k \otimes_{\mathbf{C}} \mathcal{D}\left(V_{\mathrm{reg}}\right) \rtimes W$ which maps $\xi \in V$ to $T_{\xi}$.

The map $i$ induces an algebra isomorphism $\mathrm{A}_{\text {reg }} \stackrel{\sim}{\rightarrow} k \otimes_{\mathbf{C}} \mathcal{D}\left(V_{\text {reg }}\right) \rtimes W$.

5.2.2. We consider $M=\operatorname{Ind}_{B W}^{\mathrm{A}} X=\mathrm{P} \otimes X$, where $X$ is locally nilpotent and finitely generated as an $\beta$-module, free over $k$. The action of $\xi \in V$ on $p \otimes v, p \in \mathrm{P}$ and $v \in X$ is given by

$$
\xi(p \otimes v)=p \otimes \xi v+\partial_{\xi}(p) \otimes v+\sum_{H} \sum_{0 \leq i, j \leq e_{H}-1} e_{H}\left(k_{H, i+j}-k_{H, j}\right) \frac{\alpha_{H}(\xi)}{\alpha_{H}} \varepsilon_{H, i}(p) \otimes \varepsilon_{H, j}(v) .
$$

Using Dunkl operators, i.e., via the isomorphism of Theorem 5.6, we have a structure of $W$ equivariant $\left(k \otimes_{\mathbf{C}} \mathcal{D}\left(V_{\text {reg }}\right)\right)$-module on $M_{\text {reg }}$. The corresponding connection is given by

$$
\partial_{\xi}=\xi-\sum_{H} \frac{\alpha_{H}(\xi)}{\alpha_{H}} \cdot\left(\sum_{0 \leq i, j \leq e_{H}-1} e_{H} k_{H, i+j} \varepsilon_{H, i} \otimes \varepsilon_{H, j}\right) .
$$


Hence

$$
\partial_{\xi}(p \otimes v)=p \otimes \xi v+\partial_{\xi}(p) \otimes v-\sum_{H} \sum_{i} e_{H} k_{H, i} \frac{\alpha_{H}(\xi)}{\alpha_{H}} p \otimes \varepsilon_{H, i}(v) .
$$

For the rest of 95 , we assume that $k=\mathbf{C}$.

The following result is well-known to experts, but we could not find an appropriate reference in the literature.

Proposition 5.7. The above formula for $\partial_{\xi}$ defines a $W$-equivariant integrable algebraic connection on $M$ with regular singularities.

Proof. All the claims follow from the construction, with the exception of the assertion that the singularities of the connection are regular. The connection has visibly only simple poles at the reflection hyperplanes, hence it suffices to prove the regularity at infinity with respect to some (hence any, see [De]) compactification of $V$.

Consider the $W$-equivariant compactification $Y=\mathbb{P}(\mathbf{C}+V)$ of $V$, and extend $M$ to the free $\mathcal{O}_{Y}$-module $M_{Y}:=\mathcal{O}_{Y} \otimes X$. Using a filtration of $X$ we can reduce to the case where $X=E$ is simple.

A straightforward computation shows that with respect to the extension $M_{Y}$ of $M$ and with respect to any standard coordinate patch on $Y$, the poles at infinity are also simple in this case.

5.2.3. We define a morphism of abelian groups $r: K_{0}(\mathcal{O}) \rightarrow \mathbf{Z}$ by $r([\Delta(E)])=\operatorname{dim} E$, for $E \in \operatorname{Irr}(\mathbf{C} W)$.

Lemma 5.8. Let $M \in \mathcal{O}$. Then, $M_{\mathrm{reg}}$ is a vector bundle of rank $r([M])$ on $V_{\mathrm{reg}}$.

Proof. Since $M_{\text {reg }}$ is a finitely generated $\mathbf{C}\left[V_{\text {reg }}\right]$-module with a connection, it is a vector bundle.

Now, taking the rank of that vector bundle induces a morphism $K_{0}(\mathcal{O}) \rightarrow \mathbf{Z}$, which takes the correct value on $\Delta(E)$.

\section{2 .4 .}

Proposition 5.9. Assume $k_{H, i}-k_{H, j}+\frac{i-j}{e_{H}} \notin \mathbf{Z}$, for all $H \in \mathcal{A}$ and all $0 \leq i \neq j \leq e_{H}-1$. Let $N$ be a $\Delta$-filtered object in $\mathcal{O}$. Then, for any $M \in \mathcal{O}$, we have $\operatorname{Hom}_{\mathcal{O}}(M, N) \stackrel{\sim}{\rightarrow} \operatorname{Hom}_{\overline{\mathcal{O}}}(\bar{M}, \bar{N})$.

Proof. Assume first that $M$ is also a $\Delta$-filtered object. Then, we can write $M=\operatorname{Ind}_{\beta W}^{\mathrm{A}} X$ and $N=\operatorname{Ind}_{\beta W}^{\mathrm{A}} Y$ with $X, Y$ finite dimensional $\beta W$-modules, nilpotent over $\beta$. The space $\operatorname{Hom}_{\mathrm{A}}(M, N)$ is the intersection of $\operatorname{Hom}_{\mathrm{P}}(M, N)=\mathrm{P} \otimes \operatorname{Hom}_{k}(X, Y)$ with $\operatorname{Hom}_{\mathrm{A}_{\mathrm{reg}}}\left(M_{\mathrm{reg}}, N_{\mathrm{reg}}\right)$. As in the proof of Theorem [5.3. we have to show that any element $\Psi$ of $\operatorname{Hom}_{\mathrm{P}_{\text {reg }}}\left(M_{\text {reg }}, N_{\text {reg }}\right)$ that commutes with the action of $\mathrm{A}_{\text {reg }}$ extends to a P-morphism $M \rightarrow N$. Observe that $\Psi$ is nothing but a flat, $W$-invariant section of the connection on $\operatorname{Hom}_{\mathrm{A}_{\text {reg }}}\left(M_{\text {reg }}, N_{\text {reg }}\right)$.

The residue of this connection on a hyperplane $H \in \mathcal{A}$ is constant, and has eigenvalue

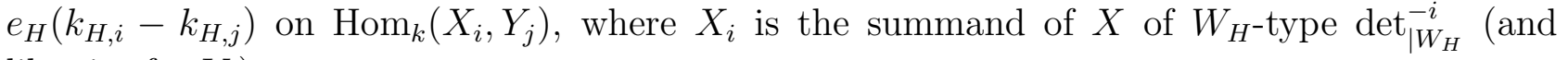
likewise for $Y_{j}$ ).

Locally near a generic point $p$ of $H$ we expand $\Psi=\sum_{l \geq l_{0}} \alpha_{H}^{l} \Psi_{l}$ with $\Psi_{l}$ holomorphic on $H$ near $p$, of $W_{H}$-type $\operatorname{det}_{\mid W_{H}}^{l}$, and with $\Psi_{l_{0}}$ not identically zero on $H$. From the lowest order term of the equation $\partial_{v_{H}}(\Psi)=0$ we see that there exist $i, j$ such that $i-j=l_{0} \bmod \left(e_{H} \mathbf{Z}\right)$, and such that $l_{0}+e_{H}\left(k_{H, i}-k_{H, j}\right)=0$. Thus $l_{0}=0$ and $\Psi$ is regular on $H$. This completes the proof of the Proposition in the special case where both $M$ and $N$ are $\Delta$-filtered. 
The general case follows from the special case above by repeating the part of the argument from the proof of Theorem [5.3, starting with formula (9).

Remark 5.10. The condition of the Proposition is equivalent to the semi-simplicity of the Hecke algebra $\mathcal{H}\left(W_{H}\right)$ of $W_{H}$. One could conjecture that this assumption can be replaced by the assumption that $\mathrm{KZ}(N)$ is a projective $\mathcal{H}\left(W_{H}\right)$-module (this would still not cover completely Theorem [5.3).

Remark 5.11. If $e_{H}=2$ for all $H$, then the condition of the Proposition reads: $k_{H} \notin \frac{1}{2}+\mathbf{Z}$.

5.2.5. Let $\mathbf{C}\left[\left(\mathbf{k}_{H, i}\right)_{1 \leq i \leq e_{H}-1}\right]$ be the polynomial ring in the indeterminates $\mathbf{k}_{H, i}$ with $\mathbf{k}_{w(H), i}=$ $\mathbf{k}_{H, i}$ for $w \in W$. We have a canonical morphism of $\mathbf{C}$-algebras $\mathbf{C}\left[\left(\mathbf{k}_{H, i}\right)\right] \rightarrow \mathbf{C}, \mathbf{k}_{H, i} \mapsto k_{H, i}$. Let $\mathfrak{m}$ be the kernel of that morphism and $R$ the completion of $\mathbf{C}\left[\left(\mathbf{k}_{H, i}\right)\right]$ at the maximal ideal $\mathfrak{m}$.

Fix $x_{0} \in V_{\text {reg }}$, and let $B_{W}=\pi_{1}\left(V_{\text {reg }} / W, x_{0}\right)$ be the Artin braid group associated to $W$.

Let $\mathcal{H}_{R}=\mathcal{H}_{R}(W, V, \gamma)$ be the Hecke algebra of $W$ over $R$, that is the quotient of $R\left[B_{W}\right]$ by the relations

$$
(T-1) \prod_{j=1}^{e_{H}-1}\left(T-\operatorname{det}(s)^{-j} \cdot e^{2 i \pi \mathbf{k}_{H, j}}\right)=0
$$

for $H \in \mathcal{A}, s \in W$ the reflection around $H$ with non-trivial eigenvalue $e^{2 i \pi / e_{H}}$ and $T$ an $s^{-}$ generator of the monodromy around $H$, cf [BrMaRou, $\S 4 . \mathrm{C}$ ]. Note that the parameters differ from [BrMaRou] because we will be using the horizontal sections functor instead of the solution functor.

We put $\mathcal{H}_{K}=\mathcal{H}_{R} \otimes_{R} K$, where $K$ is the field of fractions of $R$ and $\mathcal{H}=\mathcal{H}_{R} \otimes_{R}(R / \mathfrak{m})$.

Remark 5.12. It is known that $\mathcal{H}_{R}$ is free of rank $|W|$ over $R$ for all $W$ that do not have an irreducible component of type $G_{17 \ldots 19}, G_{24 \ldots 27}, G_{29}, G_{31 \ldots 34}$ in Shephard-Todd notation (in these cases, the statement is conjectural) $[\mathrm{Mu}$.

5.3. The Knizhnik-Zamolodchikov functor. Let $M$ be a $\left(\mathbf{C}\left[V_{\text {reg }}\right] \rtimes W\right)$-module, free of finite rank over $\mathrm{P}_{\text {reg }}=\mathbf{C}\left[V_{\text {reg }}\right]$. Let $\nabla: M \rightarrow M \otimes_{\mathbf{C}} R$ be an $R$-linear integrable connection. Then, the horizontal sections of $\nabla$ define, via the monodromy representation, an $R B_{W}$-module $L$, free over $R$.

Let $\nabla_{0}: M \rightarrow M$ be the special fiber of $\nabla$. Then, the horizontal sections of $\nabla_{0}$ is the $\mathrm{C} B_{W}$-module $L \otimes_{R}(R / \mathfrak{m})$.

Let $\nabla_{K}: M \rightarrow K \otimes_{\mathbf{C}} M$ be the generic fiber of $\nabla$. Then, the horizontal sections of $\nabla_{K}$ is the $K B_{W}$-module $L \otimes_{R} K$.

Taking horizontal sections defines an exact functor from the category of $W$-equivariant vector bundles on $R \otimes_{\mathbf{C}} V_{\text {reg }}$ with an integrable connection to the category of $R B_{W}$-modules that are free of finite rank over $R$.

Since the connection on $\Delta\left(R \otimes_{\mathbf{C}} E\right)_{\text {reg }}$ has regular singularities it follows that the connection on $M_{\text {reg }}$ has regular singularities for any $M \in \mathcal{O}_{R}^{\Delta}$.

Composing with the localisation functor, we obtain an exact functor $\mathrm{KZ}_{R}$ from $\mathcal{O}_{R}^{\Delta}$ to the category of $R B_{W}$-modules that are free of finite rank over $R$.

Similarly, we obtain exact functors $\mathrm{KZ}: \mathcal{O} \rightarrow \mathbf{C} B_{W}$-mod and $\mathrm{KZ}_{K}: \mathcal{O}_{K} \rightarrow K B_{W}$-mod.

It is well-known (cf. e.g. BrMaRou, Theorem 4.12]) that the representation of $K B_{W}$ on $\mathrm{KZ}_{K}\left(\Delta\left(K \otimes_{\mathbf{C}} E\right)\right)$ factors through $\mathcal{H}_{K}$ to give a representation corresponding (via Tits' deformation Theorem) to the representation $E$ of $\mathbf{C} W$. Recall that $\mathcal{H}=\mathcal{H}_{R} \otimes_{R}(R / \mathfrak{m})$. 
Theorem 5.13 (Hecke algebra action). The functor $\mathrm{KZ}: \mathcal{O} \rightarrow \mathbf{C} B_{W}$-mod factors through a functor $\mathrm{KZ}: \mathcal{O} / \mathcal{O}_{\text {tor }} \rightarrow \mathcal{H}$-mod. Similarly, the functor $\mathrm{KZ}_{K}: \mathcal{O}_{K} \rightarrow K B_{W}$-mod factors through a functor $\mathrm{KZ}_{K}: \mathcal{O}_{K} /\left(\mathcal{O}_{K}\right)_{\text {tor }} \rightarrow \mathcal{H}_{K}$-mod.

For $M \in \mathcal{O}_{R}^{\Delta}$, the action of $R B_{W}$ on $\mathrm{KZ}_{R}(M)$ factors through $\mathcal{H}_{R}$.

We have a commutative diagram

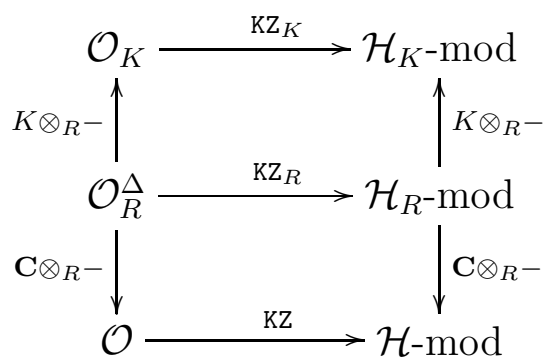

Proof. First, $\mathcal{O}_{\text {tor }}$ (and $\left.\left(\mathcal{O}_{K}\right)_{\text {tor }}\right)$ are the kernels of localisation.

When $M=\Delta\left(K \otimes_{\mathbf{C}} E\right)$, then, we have the Knizhnik-Zamolodchikov connection and the representation $\mathrm{KZ}_{K}(M)$ factors through $\mathcal{H}_{K}$. Since $\mathcal{O}_{K}$ is semi-simple (Corollary 2.20), it follows that the action on $\mathrm{KZ}_{K}(M)$ factors through $\mathcal{H}_{K}$ for any $M$ in $\mathcal{O}_{K}$.

We now consider the case of a $\Delta$-filtered module $M$ of $\mathcal{O}_{R}$. We know that the action of $K B_{W}$ on $K \otimes_{R} \mathrm{KZ}_{R}(M) \simeq \mathrm{KZ}_{K}\left(K \otimes_{R} M\right)$ factors through $\mathcal{H}_{K}$. Since $\mathrm{KZ}_{R}(M)$ is free over $R$, it follows that the action of $R B_{W}$ on $\mathrm{KZ}_{R}(M)$ factors through $\mathcal{H}_{R}$.

From this result, we deduce that the action of $\mathbf{C} B_{W}$ on $\mathrm{KZ}\left(\Delta^{r}(\mathbf{C} W)\right) \stackrel{\sim}{\rightarrow} \mathbf{C} \otimes_{R} \mathrm{KZ}_{R}\left(\Delta^{r}(R W)\right)$ factors through $\mathcal{H}$. Since every indecomposable projective object of $\mathcal{O}$ is a direct summand of $\Delta^{r}(\mathbf{C W})$ for appropriate $r$ (Corollary 2.7), it follows that the action of $\mathbf{C} B_{W}$ on $\operatorname{KZ}(M)$ factors through $\mathcal{H}$ for every projective $M$, hence for every $M$ in $\mathcal{O}$.

5.4. Main results. In this subsection we assume that $\operatorname{dim} \mathcal{H}=|W|$, cf. Remark [5.12,

The functor $\mathrm{KZ}: \mathcal{O} \rightarrow \mathcal{H}$-mod is exact. Hence, it is represented by a projective $P_{\mathrm{KZ}} \in \mathcal{O}$. In other words, there exists an algebra morphism $\phi: \mathcal{H} \rightarrow\left(\operatorname{End}_{\mathcal{O}} P_{\mathrm{KZ}}\right)^{\text {opp }}$ such that the functor $\mathrm{KZ}$ is isomorphic to $\operatorname{Hom}_{\mathcal{O}}\left(P_{\mathrm{KZ}},-\right)$.

We know also, see 5 5.1.1, that the functor KZ factors through $\mathcal{O} / \mathcal{O}_{\text {tor }} \rightarrow \mathcal{H}$-mod.

Theorem 5.14. The functor $\mathrm{KZ}$ induces an equivalence: $\mathcal{O} / \mathcal{O}_{\text {tor }} \stackrel{\sim}{\rightarrow} \mathcal{H}$-mod.

This Theorem is equivalent to

Theorem 5.15. The morphism $\phi: \mathcal{H} \rightarrow\left(\text { End }_{\mathcal{O}} P_{\mathrm{Kz}}\right)^{\mathrm{opp}}$ is an algebra isomorphism.

Proof of Theorems 5.14 5.15. Recall that the horizontal sections functor gives an equivalence from the category of vector bundles over $V_{\text {reg }} / W$ with a regular integrable connection to the category of finite-dimensional $\mathbf{C} B_{W}$-modules (Riemann-Hilbert correspondence, De, Theorems I.2.17 and II.5.9]).

We deduce from $\$ 5.1 .1$ that $\mathrm{KZ}: \mathcal{O} / \mathcal{O}_{\text {tor }} \rightarrow \mathcal{H}$ is a fully faithful exact functor with image a full subcategory closed under taking subobjects and quotients. Furthermore, $\overline{P_{\mathrm{KZ}}}$, the image of $P_{\mathrm{Kz}}$ in $\mathcal{O} / \mathcal{O}_{\text {tor }}$, is a progenerator of $\mathcal{O} / \mathcal{O}_{\text {tor }}$. Thus, Theorem 5.14 follows from Theorem 5.15 ,

To prove Theorem [5.15, observe that the morphism $\phi$ is surjective. Indeed, let $\mathcal{C}^{\prime}$ be a full subcategory of an abelian category $\mathcal{C}$, closed under taking quotients, and ${ }^{\top} F$ a left adjoint to the inclusion $F: \mathcal{C}^{\prime} \hookrightarrow \mathcal{C}$. Then the adjunction morphism $\eta: \operatorname{Id}_{\mathcal{C}} \rightarrow F \circ\left({ }^{\top} F\right)$ is surjective, since 
$\eta(X)$ is the canonical map from $X$ to its largest quotient in $\mathcal{C}^{\prime}$. This proves surjectivity of the morphism $\phi$ above.

Further, we have

$$
P_{\mathrm{KZ}}=\bigoplus_{E \in \operatorname{Irr}(\mathbf{C} W)}(\operatorname{dim} \mathrm{KZ}(L(E))) P(E)
$$

Hence, we compute

$$
\begin{gathered}
\operatorname{dim}\left(\operatorname{End}_{\mathcal{O}} P_{\mathrm{KZ}}\right)=\bigoplus_{E, F} \operatorname{dim} \mathrm{KZ}(L(E)) \operatorname{dim} \mathrm{KZ}(L(F)) \operatorname{dim} \operatorname{Hom}(P(E), P(F)) \\
=\bigoplus_{E, F, G} \operatorname{dim} \mathrm{KZ}(L(E)) \operatorname{dim} \mathrm{KZ}(L(F))[P(E): \Delta(G)][\Delta(G): L(F)] \\
=\bigoplus_{E, F, G} \operatorname{dimKZ}(L(E)) \operatorname{dimKZ}(L(F))[\nabla(G): L(E)][\Delta(G): L(F)] \\
=\bigoplus_{G} \operatorname{dimKZ}(\nabla(G)) \operatorname{dimKZ}(\Delta(G))
\end{gathered}
$$

Now, the restrictions of $\nabla(G)$ and $\Delta(G)$ to $V_{\text {reg }}$ are vector bundles of rank $\operatorname{dim} G$ (Proposition 3.3 and Lemma [5.8), hence $\operatorname{dim}\left(\operatorname{End}_{\mathcal{O}} P_{\mathrm{KZ}}\right)=|W|=\operatorname{dim} \mathcal{H}$. This shows that $\phi$ is an isomorphism. Note that this rank computation can also be achieved by deformation to $R$.

The following result shows that the category $\mathcal{O}$ can be completely recovered from $\mathcal{H}$ and a certain $\mathcal{H}$-module :

Theorem 5.16 (Double-centralizer property). Let $Q$ be a projective in $\mathcal{O}$. Then, the canonical map $\operatorname{Hom}_{\mathcal{O}}(M, Q) \rightarrow \operatorname{Hom}_{\mathcal{H}}(\mathrm{KZ}(M), \mathrm{KZ}(Q))$ is an isomorphism, for any $M \in \mathcal{O}$.

Furthermore, if $X$ is a progenerator for $\mathcal{O}$, then, we have an equivalence

$$
\left(\operatorname{End}_{\mathcal{H}} \mathrm{KZ}(X)\right)^{\text {opp }} \text {-mod } \stackrel{\sim}{\rightarrow} \mathcal{O} \text {. }
$$

Proof. The first part follows from Theorems 5.3 and 5.15] and the second from Corollary 5.5]

Remark 5.17. We conjecture that, if $W=S_{n}$, then $\mathcal{O}$ is equivalent to the category of finitelygenerated modules over the associated $q$-Schur algebra. That would imply, in particular, that if $k_{H, 1}=k_{1}<0$ is a negative real constant, then the Cherednik algebras $\mathrm{A}\left(S_{n}\right)$ with parameters $k_{1}$ and $k_{1}-1$, respectively, are Morita equivalent.

Let $Z(\mathcal{H})$ denote the center of the algebra $\mathcal{H}$ and $Z(\mathcal{O})$ the center of category $\mathcal{O}$ (i.e. the algebra of endomorphisms of the identity functor $\left.\operatorname{Id}_{\mathcal{O}}\right)$.

Corollary 5.18. The canonical morphism $Z(\mathcal{O}) \rightarrow \operatorname{End}_{\mathcal{O}} P_{\mathrm{KZ}}$ induces an isomorphism $Z(\mathcal{O}) \stackrel{\sim}{\rightarrow}$ $Z(\mathcal{H})$. In particular, the functor $\mathrm{KZ}$ induces a bijection between blocks of $\mathcal{O}$ and blocks of $\mathcal{H}$.

Proof. This follows immediately from Theorem [5.16] given two rings $B$ and $C$ and a $(B, C)$ bimodule $M$ such that the canonical morphisms $B \stackrel{\sim}{\rightarrow} \operatorname{End}_{C \text { opp }}(M)$ and $C \stackrel{\sim}{\rightarrow}\left(\operatorname{End}_{B} M\right)^{\text {opp }}$ are isomorphisms, then we have a canonical isomorphism $Z(B) \stackrel{\sim}{\rightarrow} Z(C)$.

The decomposition matrix $K_{0}\left(\mathcal{O}_{K}\right) \rightarrow K_{0}(\mathcal{O})$ is triangular. We deduce the triangularity of decomposition matrices of Hecke algebras, in characteristic 0 :

Corollary 5.19. The decomposition matrix $K_{0}\left(\mathcal{H}_{K}\right) \rightarrow K_{0}(\mathcal{H})$ is triangular. 
5.4.1. KZ-functor and Twist. Let $\zeta$ be a one-dimensional character of $W$ and $\tau_{\zeta}: \mathbf{C} W \stackrel{\sim}{\rightarrow} \mathbf{C} W$ the automorphism given by $w \mapsto \zeta(w) \cdot w$ for $w \in W$. This extends to an isomorphism

$$
\tau_{\zeta}: A(\gamma) \stackrel{\sim}{\rightarrow} A\left(\tau_{\zeta}(\gamma)\right), \quad V \ni \xi \mapsto \xi, \quad V^{*} \ni x \mapsto x, \quad W \ni w \mapsto \zeta(w) \cdot w .
$$

We obtain an equivalence $\mathcal{O}(\gamma) \stackrel{\sim}{\rightarrow} \mathcal{O}\left(\tau_{\zeta}(\gamma)\right)$, sending $V(E)$ to $V\left(E \otimes \zeta^{-1}\right)$, where $V$ stands for any of the symbols: $L, \Delta, \nabla, P, I, T$.

For $H \in \mathcal{A}$, let $d_{H} \in\left\{1, \ldots, e_{H}\right\}$ such that $\zeta_{\mid W_{H}}=\operatorname{det}_{\mid W_{H}}^{d_{H}}$. Define an automorphism $\eta_{\zeta}$ of $\mathcal{D}\left(V_{\text {reg }}\right) \rtimes W$ by

$$
\begin{gathered}
P \ni f \mapsto f, \quad W \ni w \mapsto \zeta(w) \cdot w \\
\text { and } \partial_{\xi} \mapsto \partial_{\xi}-\sum_{H} \frac{\alpha_{H}(\xi)}{\alpha_{H}} \varepsilon_{H, e_{H}} \cdot e_{H} \cdot k_{H, e_{H}-d_{H}} \text { for } \xi \in V
\end{gathered}
$$

(for notation, see Remark 3.2). We have a commutative diagram

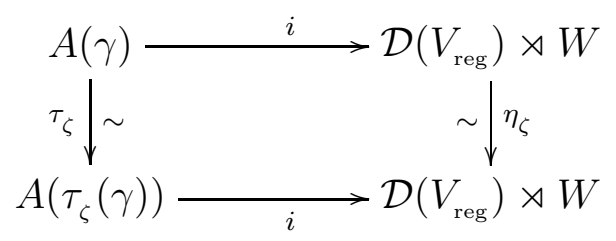

Given $M$ a $\left(\mathcal{D}\left(V_{\text {reg }}\right) \rtimes W\right)$-module, then $\left(\eta_{\zeta}\right)_{*} M \stackrel{\sim}{\rightarrow} M \otimes_{\mathcal{O}_{V_{\text {reg }}}} \Delta\left(\zeta^{-1}\right)_{\text {reg }}$.

This self-equivalence of the category of $W$-equivariant bundles with a regular singular connection on $V_{r e g}$ corresponds, via the horizontal sections functor, to the automorphism of $\mathbf{C} B_{W}$ given by

$$
T \mapsto e^{-2 i \pi k_{H, e_{H}-d_{H}} \zeta(s)^{-1} T}
$$

for $H \in \mathcal{A}, s \in W$ the reflection around $H$ with non-trivial eigenvalue $e^{2 i \pi / e_{H}}$ and $T$ an $s^{-}$ generator of the monodromy around $H$. This induces an isomorphism $\mathcal{H}(\zeta): \mathcal{H}(W, \gamma) \stackrel{\sim}{\rightarrow}$ $\mathcal{H}\left(W, \tau_{\zeta}(\gamma)\right)$ and the following diagram is commutative :

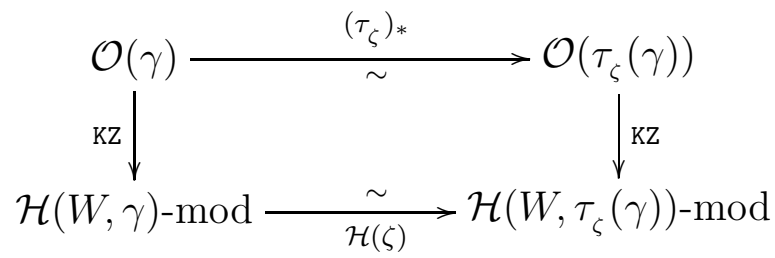

5.4.2. KZ-functor and Duality. We have a commutative diagram

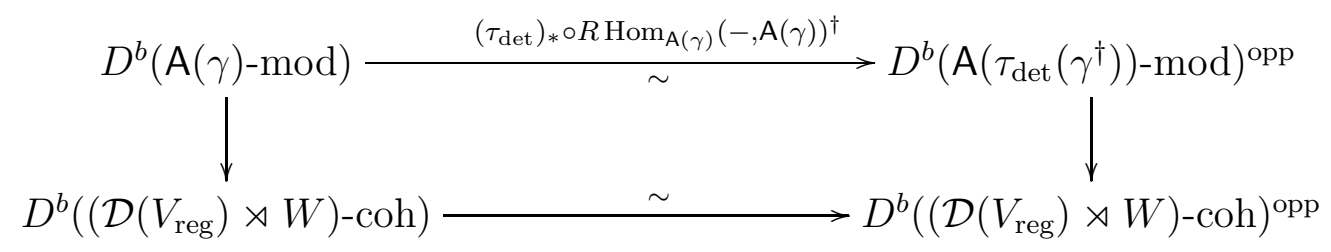

where the vertical arrows are given by localisation followed by the Dunkl operator isomorphism $i$ of Theorem 5.6 and the bottom horizontal arrow is the classical $\mathcal{D}$-module duality.

Consider the isomorphism $\mathbf{C} B_{W} \stackrel{\sim}{\rightarrow}\left(\mathbf{C} B_{W}\right)^{\text {opp }}$ given by $T \mapsto \operatorname{det}(s)^{-1} e^{2 i \pi k_{H, 1}} T^{-1}$ 
for $H \in \mathcal{A}, s \in W$ the reflection around $H$ with non-trivial eigenvalue $e^{2 i \pi / e_{H}}$ and $T$ an $s$-generator of the monodromy around $H$. It induces an isomorphism

$$
\mathcal{H}\left({ }^{\dagger}\right): \mathcal{H}(W, \gamma) \stackrel{\sim}{\rightarrow} \mathcal{H}\left(W, \gamma^{\dagger}\right)^{\text {opp }}
$$

We conclude that we have a commutative diagram

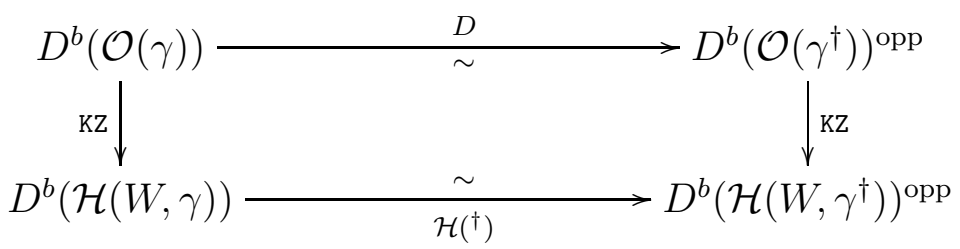

On the other hand, by Lemma 5.2, we know that $(-)^{\vee}$ preserves $\mathcal{O}_{\text {tor }}$, hence descends to the quotient category $\mathcal{O} / \mathcal{O}_{\text {tor }}$, i.e., there is an equivalence $\Phi$ making the following diagram commute:

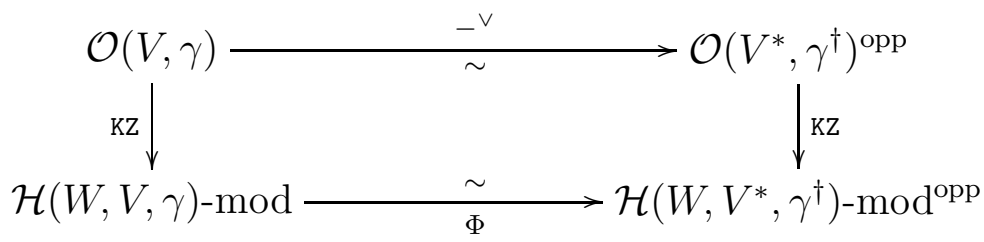

Further, choose a $W$-invariant hermitian form on $V$, i.e., a semi-linear $W$-equivariant isomorphism $\kappa: V \stackrel{\sim}{\rightarrow} V^{*}$. Then, we get an isomorphism $\pi_{1}\left(V_{\text {reg }} / W, x_{0}\right) \stackrel{\sim}{\rightarrow} \pi_{1}\left(V_{\text {reg }}^{*} / W, \kappa\left(x_{0}\right)\right)$. It induces an isomorphism $\mathcal{H}(\kappa): \mathcal{H}(W, V, \gamma) \stackrel{\sim}{\rightarrow} \mathcal{H}\left(W, V^{*}, \gamma\right)$. Composing with $\mathcal{H}\left({ }^{\dagger}\right)$, we obtain an isomorphism $\mathcal{H}\left(\kappa \circ(-)^{\dagger}\right): \mathcal{H}(W, V, \gamma) \stackrel{\sim}{\rightarrow} \mathcal{H}\left(W, V^{*}, \gamma^{\dagger}\right)^{\text {opp}}$, which we denote below by $\psi$.

Remark 5.20. One could conjecture that the two functors $\Phi$ and $\psi_{*}$ are isomorphic (they induce the same maps at the level of Grothendieck groups).

5.4.3. The A-module $P_{\mathrm{Kz}}$ and Duality. Let $\operatorname{Irr}(W, V, \gamma) \subset \operatorname{Irr}(W)$ denote the subset formed by all $E \in \operatorname{Irr}(W)$ such that $L(E)_{\text {reg }} \neq 0$. We have a bijection $\operatorname{Irr}(W, V, \gamma) \stackrel{\sim}{\rightarrow} \operatorname{Irr}\left(W, V^{*}, \gamma^{\dagger}\right), E \mapsto E^{\vee}$ (Proposition 4.7 and Lemma [5.2). Thus, $P_{\mathrm{KZ}}=\bigoplus_{E \in \operatorname{Irr}(W, V, \gamma)}(\operatorname{dim} \mathrm{KZ}(L(E))) \cdot P(E)$.

To make the dependence on $V$ and $\gamma$ explicit, we will write $P_{\mathrm{KZ}}=P_{\mathrm{Kz}}(V, \gamma)$.

Proposition 5.21. (i) We have $D\left(P_{\mathrm{KZ}}(V, \gamma)\right) \simeq P_{\mathrm{KZ}}\left(V, \gamma^{\dagger}\right)$ and $P_{\mathrm{KZ}}(V, \gamma)^{\vee} \simeq P_{\mathrm{KZ}}\left(V^{*}, \gamma\right)$. In particular, $P_{\mathrm{Kz}}$ is both projective and injective.

(ii) For $E \in \operatorname{Irr}(W)$, the following are equivalent

- $E \in \operatorname{Irr}(W, V, \gamma)$

- $L(E)$ is a submodule of a standard module

- $P(E)$ is a submodule of $P_{\mathrm{KZ}}$

- $P(E)$ is injective

- $P(E)$ is tilting

- $I(E)$ is projective

- $I(E)$ is tilting

Proof. The first claim follows from $\$ 5.4 .2$ Proposition 4.7 then implies that $P_{\mathrm{Kz}}$ is injective.

The considerations above imply that if $E \in \operatorname{Irr}(W, V, \gamma)$, then $P(E)$ is injective and tilting. The assertions about $I(E)$ follow by applying $(-)^{\vee}$.

We know that if $L(E)$ is a submodule of a $\Delta$-filtered module or a quotient of a $\nabla$-filtered module, then $E \in \operatorname{Irr}(W, V, \gamma)$. 
This shows that any of the assertions about $P(E)$ or $I(E)$ implies that $E \in \operatorname{Irr}(W, V, \gamma)$.

\section{Relation to Kazhdan-Lusztig theory of Cells}

We review some parts of Kazhdan-Lusztig and Lusztig's theory of Weyl group representations.

\subsection{Lusztig's algebra $\mathcal{J}$.}

6.1.1. Let $(W, S)$ be a finite Weyl group, $\mathcal{H}$ be its Hecke algebra, a $\mathbf{Z}\left[v, v^{-1}\right]$-algebra with basis $\left\{T_{w}\right\}_{w \in W}$ and relations

$$
T_{w} T_{w^{\prime}}=T_{w w^{\prime}} \text { if } l\left(w w^{\prime}\right)=l(w)+l\left(w^{\prime}\right) \quad \text { and } \quad\left(T_{s}+1\right)\left(T_{s}-v^{2}\right)=0 \text { for } s \in S .
$$

Lusztig associated to $W$ a Z-ring $\mathcal{J}$, usually referred to as asymptotic Hecke algebra, Lu3, $\S 2.3]$. Let $\varpi: \mathcal{H} \rightarrow \mathbf{Z}\left[v, v^{-1}\right] \otimes_{\mathbf{Z}} \mathcal{J}$ be Lusztig's morphism of $\mathbf{Z}\left[v, v^{-1}\right]$-algebras [Lu3, §2.4].

The ring $\mathbf{Q} \otimes_{\mathbf{Z}} \mathcal{J}$ is semi-simple and the morphism $\operatorname{Id}_{\mathbf{Q}(v)} \otimes \varpi$ is an isomorphism.

For any commutative $\mathbf{Q}\left[v, v^{-1}\right]$-algebra $R$ we put $\mathcal{H}_{R}:=R \otimes \mathbf{z}\left[v, v^{-1}\right] \mathcal{H}$.

Definition 6.1. The $\mathcal{H}_{R}$-modules $S(M)=\varpi^{*}\left(R \otimes_{\mathbf{Q}} M\right)$, for $M$ a simple $\mathbf{Q} \otimes_{\mathbf{z}} \mathcal{J}$-module, will be referred to as standard $\mathcal{H}_{R}$-modules. ${ }^{3}$

When $R=\mathbf{Q}(v)$, then the standard $\mathcal{H}_{R}$-modules are simple and this gives a bijection from the set of simple $\left(\mathbf{Q} \otimes_{\mathbf{z}} \mathcal{J}\right)$-modules to the set of simple $\left(\mathbf{Q}(v) \otimes_{\mathbf{Z}\left[v, v^{-1}\right]} \mathcal{H}\right)$-modules.

Similarly, taking $K=\mathbf{Q}\left[v, v^{-1}\right] /(v-1)$, we obtain a bijection from the set of simple $\left(\mathbf{Q} \otimes_{\mathbf{z}} \mathcal{J}\right)$ modules to the set of simple $\mathrm{Q} W$-modules.

We will identify these sets of simple modules via these bijections.

We have an order $\leq_{L R}$ on $W$ constructed in [KaLu, p.167]. We denote by $\mathcal{C}$ the set of two-sided cells of $W$ and by $\leq$ the order on $\mathcal{C}$ coming from $\leq_{L R}$.

Let $\left\{C_{w}\right\}_{w \in W}$ be the Kazhdan-Lusztig basis for $\mathcal{H}$. Let $I$ be an ideal of $\mathcal{C}$, i.e., a subset such that given $\underline{\mathrm{c}} \leq \underline{\mathrm{c}}^{\prime}$, then $\underline{\mathrm{c}}^{\prime} \in I \Rightarrow \underline{\mathrm{c}} \in I$. We put $\mathcal{H}^{I}=\bigoplus_{\underline{\mathrm{c}} \in I, w \in \underline{\mathrm{c}}} \mathbf{Z}\left[v, v^{-1}\right] C_{w}$. This is a two-sided ideal of $\mathcal{H}$ [Lu1, p.137].

The ring $\mathcal{J}$ comes with a $\mathbf{Z}$-basis $\left\{t_{w}\right\}_{w \in W}$ and we put $\mathcal{J}_{\underline{\mathbf{c}}}=\bigoplus_{w \in \underline{\mathrm{c}}} \mathbf{Z} t_{w}$. This is a block of $\mathcal{J}$ and $\mathcal{J}=\bigoplus_{\underline{\mathbf{c}} \in \mathcal{C}} \mathcal{J}_{\underline{\mathbf{c}}}$. The corresponding partition of the set of simple $\left(\mathbf{Q} \otimes_{\mathbf{Z}} \mathcal{J}\right)$-modules is called the partition into families.

Given $I$ an ideal of $\mathcal{C}$, we denote by $I^{\circ}$ the set of $\underline{\mathrm{c}} \in I$ such that there is $\underline{\mathrm{c}}^{\prime} \in I$ with $\underline{\mathrm{c}}<\underline{\mathrm{c}}^{\prime}$.

The following is a slight reformulation of [Lu3, §1.4] :

Proposition 6.2. Let $I$ be an ideal of $\mathcal{C}$. Then, the assignment $t_{w} \mapsto C_{w}$ induces an isomorphism of $\mathcal{H}$-modules

$$
\bigoplus_{\underline{\mathbf{c}} \in I-I^{\circ}} \varpi^{*}\left(\mathbf{Z}\left[v, v^{-1}\right] \otimes \mathbf{z} \mathcal{J}_{\underline{\mathbf{c}}}\right) \stackrel{\sim}{\rightarrow} \mathcal{H}^{I} / \mathcal{H}^{I^{\circ}}
$$

In particular, the $\left(\mathbf{Q}\left[v, v^{-1}\right] \otimes_{\mathbf{z}\left[v, v^{-1}\right]} \mathcal{H}\right)$-module $\mathbf{Q}\left[v, v^{-1}\right] \otimes_{\mathbf{Z}\left[v, v^{-1}\right]}\left(\mathcal{H}^{I} / \mathcal{H}^{I^{\circ}}\right)$ is a direct sum of standard $\mathcal{H}_{\mathbf{Q}\left[v, v^{-1}\right]}$-modules.

This proposition gives a characterization of standard $\mathcal{H}_{\mathbf{Q}\left[v, v^{-1}\right]}$-modules via the Hecke algebra filtration coming from two-sided cells.

\footnotetext{
${ }^{3}$ There seems to be no name for such modules in the literature.
} 
6.1.2. Next, we consider filtrations coming from certain functions on the set of two-sided cells.

Definition 6.3. A sorting function $f: W \rightarrow \mathbf{Z}$ is a function constant on two-sided cells and such that $\underline{\mathrm{c}}^{\prime}<\underline{\mathrm{c}} \Rightarrow f\left(\underline{\mathrm{c}}^{\prime}\right)>f(\underline{\mathrm{c}})$.

Given a sorting function $f$, we put $\mathcal{H}_{R}^{\geq i}:=\bigoplus_{w \in W, f(w) \geq i} R \cdot C_{w}$ and $\mathcal{H}_{R}^{>i}:=\bigoplus_{w \in W, f(w)>i} R \cdot C_{w}$. Then, $\mathcal{H}_{R}^{\geq i}$ is a two-sided ideal of $\mathcal{H}_{R}$, since $I=\{\underline{\mathrm{c}} \in \mathcal{C} \mid f(\underline{\mathrm{c}}) \geq i\}$ is an ideal. Similarly, $\mathcal{H}_{R}^{>i}$ is a two-sided ideal of $\mathcal{H}_{R}$. Furthermore, $I^{\circ} \subseteq\{\underline{\mathrm{c}} \in \mathcal{C} \mid f(\underline{\mathrm{c}})>i\}$. Consequently, we deduce from Proposition 6.2 :

Corollary 6.4. We have an isomorphism of $\mathcal{H}$-modules

$$
\bigoplus_{\underline{\mathrm{c}} \in \mathcal{C}, f(\underline{\mathrm{c}})=i} \varpi^{*}\left(\mathbf{Z}\left[v, v^{-1}\right] \otimes_{\mathbf{z}} \mathcal{J}_{\underline{\mathrm{c}}}\right) \stackrel{\sim}{\rightarrow} \mathcal{H}^{\geq i} / \mathcal{H}^{>i} .
$$

In particular, the $\left(\mathbf{Q}\left[v, v^{-1}\right] \otimes_{\mathbf{z}\left[v, v^{-1}\right]} \mathcal{H}\right)$-module $\mathbf{Q}\left[v, v^{-1}\right] \otimes_{\mathbf{z}\left[v, v^{-1}\right]}\left(\mathcal{H}^{\geq i} / \mathcal{H}^{>i}\right)$ is a direct sum of standard $\mathcal{H}_{\mathbf{Q}\left[v, v^{-1}\right]}$-modules.

Thus, we have another characterization of standard $\mathcal{H}_{\mathbf{Q}\left[v, v^{-1}\right]}$-modules via the Hecke algebra filtration coming from $f$.

Let $\mathcal{F}$ be the set of families of irreducible characters of $W$. We transfer the concepts associated with $\mathcal{C}$ to $\mathcal{F}$ via the canonical bijection between $\mathcal{C}$ and $\mathcal{F}$.

In particular, we have a function $f: \operatorname{Irr}(W) \rightarrow \mathbf{Z}$ constant on families.

We have $\mathcal{H}^{\geq i}=\mathcal{H} \cap\left(\bigoplus_{f(E) \geq i} e_{E} \mathbf{Q}(v) \otimes_{\mathbf{Z}\left[v, v^{-1}\right]} \mathcal{H}\right)$, where $e_{E}$ is the primitive central idempotent of $\mathbf{Q}(v) \otimes_{\mathbf{Z}\left[v, v^{-1}\right]} \mathcal{H}$ that acts as 1 on the simple $\left(\mathbf{Q}(v) \otimes_{\mathbf{Z}\left[v, v^{-1}\right]} \mathcal{H}\right)$-module corresponding to $E$.

This shows that, if $R$ is a localisation of $\mathbf{Q}\left[v, v^{-1}\right]$, then the filtration on $\mathcal{H}_{R}=R \otimes_{\mathbf{Z}\left[v, v^{-1}\right]} \mathcal{H}$ given by $f$ can be recovered without using the Kazhdan-Lusztig basis. We obtain

Proposition 6.5. Let $R$ be a localisation of $\mathbf{Q}\left[v, v^{-1}\right]$ and $P$ be a projective $\mathcal{H}_{R}$-module. Let $Q^{\geq i}$ (resp. $Q^{>i}$ ) be the sum of the simple submodules $E$ of $\mathbf{Q}(v) \otimes_{R} P$ such that $f(E) \geq i$ (resp. $f(E)>i)$.

Then, $\left(P \cap Q^{\geq i}\right) /\left(P \cap Q^{>i}\right)$ is a direct sum of standard $\mathcal{H}_{R}$-modules.

Thus, any sorting function yields a characterization of the standard $\mathcal{H}_{R^{-}}$modules without using the Kazhdan-Lusztig basis.

6.1.3. Given $E \in \operatorname{Irr}(W)$, we denote by $a_{E}$ (resp. $A_{E}$ ) the lowest (resp. highest) power of $q$ in the generic degree of $E$ [Lu1, §4.1.1].

By [Lu2, Theorem 5.4 and Corollary 6.3 (b)], Lusztig's $a$-function is a sorting function. The corresponding filtrations on projective modules have been considered in GeRou.

Write $E<E^{\prime}$ for the order on $\mathcal{F}$ arising from $<_{K L}$ via the canonical bijection between $\mathcal{C}$ and $\mathcal{F}$. The following Lemma is a classical result :

Lemma 6.6. Let $E, E^{\prime} \in \mathcal{F}$. If $E<E^{\prime}$, then $a_{E}>a_{E^{\prime}}$ and $A_{E}>A_{E^{\prime}}$.

Proof. By KaLu, Remark 3.3(a)], we have $v \leq_{L R} w$ if and only if $w_{0} w \leq_{L R} w_{0} v$, where $w_{0}$ is the element of maximal length. Left multiplication by $w_{0}$ induces a automorphism of $\mathcal{C}$. The corresponding automorphism of $\mathcal{F}$ is tensor product by det [Lu1, Lemma 5.14]. It follows that $E<E^{\prime}$ if and only if $E^{\prime} \otimes \operatorname{det}<E \otimes \operatorname{det}(\operatorname{cf}$ also [BaVo, Proposition 2.25]).

We have $A_{E}=N-a_{E \otimes \text { det }}$, where $N$ is the number of positive roots of $W$ [Lu1, 5.11.5].

The Lemma is now a consequence of the fact that $\left.E<E^{\prime} \Rightarrow a_{E}>a_{E}\right)$. 
We deduce there is another sorting function :

Proposition 6.7. The function $a_{E}+A_{E}$ is a sorting function.

\subsection{Standard modules for the Hecke algebra via KZ-functor.}

6.2.1. We consider the setting of $\$ 3.1$ with $k_{H, 1}=k_{1}$ independent of $H$. According to BrMi, $\S 4.21$ and Proposition 4.1] we have

$$
c_{E}=k_{1}\left(a_{E}+A_{E}\right) .
$$

We can finally identify the images of the standard modules $\Delta(E)$ of $\mathcal{O}$ via $\mathrm{KZ}$ :

Theorem 6.8. Assume $k_{H, 1}$ is a positive real number independent of $H$. Let $E \in \operatorname{Irr}(\mathbf{C} W)$. Then, $\mathrm{KZ}(\Delta(E)) \stackrel{\sim}{\rightarrow} S(E)$.

Proof. We prove the result for $R$ local complete as in 5.2 .5 instead of $\mathbf{C}$. The $\Delta$-filtration of projective objects of $\mathcal{O}_{R}$ becomes, via $\mathrm{KZ}_{R}$, the filtration of Proposition [6.5] for the sorting function $f(E)=a_{E}+A_{E}$ and the associated quotients are direct sums of standard $\mathcal{H}_{R^{-}}$modules. It follows that the modules $\mathrm{KZ}_{R}(\Delta(R \otimes E))$ for $E \in \operatorname{Irr}(W)$ coincide with the standard $\mathcal{H}_{R^{-}}$ modules. Since $\mathrm{KZ}_{K}(\Delta(K \otimes E)) \stackrel{\sim}{\rightarrow} K \otimes S(E)$ (cf the remark before Theorem[5.13) ), we deduce the Theorem.

Remark 6.9. If the number $k_{H, 1}$ (which is independent of $H$ ) is non-real, then the category $\mathcal{O}$ and the algebra $\mathcal{H}$ are both semi-simple, hence it is still true that $\operatorname{KZ}(\Delta(E)) \simeq S(E)$. If the number is non-positive real, then a similar approach shows that $\mathrm{KZ}(\Delta(E)) \simeq S(E)^{*}$.

Corollary 6.10. Assume $k_{H, 1}$ is a positive real number and $W$ has type $A_{n}$. Then, $\operatorname{KZ}(\Delta(E))$ is isomorphic to the Specht module corresponding to E.

Proof. The result is a consequence of [Na, GaMc, where it is proven that the module $S(E)$ is a Specht module. Alternatively, any projective $\mathcal{H}$-module is known to have a filtration by Specht modules such that the order of terms in the filtration is compatible with the dominance order on partitions. The claim of the Corollary can be easily deduced from this by comparing with the order relation on two-sided cells.

Corollary 6.11. If $\mathrm{KZ}(L(E)) \neq 0$, then $\mathrm{KZ}(P(E))$ is a projective $\mathcal{H}$-module and, for any $F \in \operatorname{Irr}(W)$, we have $[S(F): \operatorname{KZ}(L(E))]=[P(E): \Delta(F)]$.

Proof. This is an immediate consequence of Theorem 6.8, the reciprocity formula in $\$ 2.6 .2$, and Proposition 3.3 .

\section{REFERENCES}

[A] S. Arkhipov, Semi-infinite cohomology of associative algebras and bar duality, Internat. Math. Res. Notices (1997), no. 17, 833-863.

[BaVo] D. Barbasch and D. Vogan, Primitive ideals and orbital integrals in complex exceptional groups, J. of Alg. 80 (1983), 350-382.

[BeEtGi] Y. Berest, P. Etingof and V. Ginzburg, Cherednik algebras and differential operators on quasiinvariants, arXiv:math.QA/0111005, to appear in Duke Math. J.

[Bj] J.-E. Bjork, Rings of differential operators. North-Holland Mathematical Library, 21. North-Holland Publishing Co., Amsterdam-New York, 1979.

[Bo] A. Borel et al., "Algebraic D-modules", Academic Press, 1987. 
[BrMaRou] M. Broué, G. Malle and R. Rouquier, Complex reflection groups, braid groups, Hecke algebras, J. reine angew. Math. 500 (1998), 127-190.

[BrMi] M. Broué and J. Michel, Sur certains éléments réguliers des groupes de Weyl et les variétés de Deligne-Lusztig associées, in "Finite reductive groups", Birkhäuser, 73-139, 1997.

[CPS1] E. Cline, B. Parshall and L. Scott, Finite dimensional algebras and highest weight categories, J. reine angew. Math. 391 (1988), 85-99.

[CPS2] E. Cline, B. Parshall and L. Scott, "Stratifying endomorphism algebras", Mem. Amer. Math. Soc. 124 (1996).

[De] P. Deligne, "Equations différentielles à points singuliers réguliers", Lecture Notes in Math. 163, Springer Verlag, 1970.

[DJ] R. Dipper, G. James, Representations of Hecke Algebras of General Linear Groups, Proc. London Math. Soc. 52 (1986), 20-52.

[DuOp] C. Dunkl and E. Opdam, Dunkl operators for complex reflection groups, Proc. London Math. Soc. 86 (2003), 70-108.

[EtGi] P. Etingof and V. Ginzburg, Symplectic reflection algebras, Calogero-Moser space and deformed Harish-Chandra homomorphism, Inv. Math. 147 (2002), 243-348.

[GaMc] A. M. Garsia and T. J. McLarnan, Relations between Young's natural and the Kazhdan-Lusztig representations of $S_{n}$., Adv. in Math. 69 (1988), 32-92.

[GeRou] M. Geck and R. Rouquier, Filtrations on projective modules for Iwahori-Hecke algebras, in "Modular representation theory of finite groups", de Gruyter, 211-221, 2001.

$[\mathrm{Gu}] \quad$ N. Guay, Projective modules in the category $\mathcal{O}$ for the Cherednik algebra, preprint (December 2001).

$[\mathrm{KaLu}] \quad$ D. Kazhdan and G. Lusztig, Representations of Coxeter groups and Hecke algebras, Inv. Math. 53 (1979), 165-184.

[Lu1] G. Lusztig, "Characters of reductive groups over a finite field", Ann. of Math. Studies, vol. 107, Princeton Univ. Press, 1984.

[Lu2] G. Lusztig, Cells in affine Weyl groups, in "Algebraic groups and related topics", Adv. Studies in Pure Math. Vol. 6, Kinokuniya and North-Holland, 1985, pp. 255-287.

[Lu3] G. Lusztig, Cells in affine Weyl groups, III, J. Fac. Sci. Univ. Tokyo Sect. IA, Math. 34 (1987), 223-243.

[Mu $\quad$ J. Müller, letter, 25 October 2002.

[Na] H. Naruse, On an isomorphism between Specht module and left cell of $\mathfrak{S}_{n}$, Tokyo J. Math. 12 (1989), 247-267.

[Ri] C.M. Ringel, The category of modules with good filtrations over a quasi-hereditary algebra has almost split sequences, Math. Z. 208 (1991), 209-223.

[So1] W. Soergel, Kategorie $\mathcal{O}$, perverse Garben und Moduln über den Koinvarianten zur Weylgruppe, Journal of the A.M.S. 3 (1990), 421-445.

[So2] W. Soergel, Charakterformeln für Kipp-Moduln über Kac-Moody Algebren, Representation Theory 1 (1997), 115-132.

V. Ginzburg: Department of Mathematics, University of Chicago, Chicago, IL 60637, USA; ginzburg@math .uchicago.edu

N. Guay: Department of Mathematics, University of Chicago, Chicago, IL 60637, USA; nguay@math . uchicago .edu

E. Opdam: Korteweg de Vries Institute for Mathematics, University of Amsterdam,

Plantage Muidergracht 24, 1018TV Amsterdam, The Netherlands; opdam@science.uva.nl

R. Rouquier: UFR de Mathématiques et Institut de Mathématiques de Jussieu (CNRS UMR 7586), Université Paris VII, 2 place Jussieu, 75251 Paris Cedex 05, France; rouquier@math.jussieu.fr 